Molecules 2006, 11, 81-102

molecules

ISSN 1420-3049

http://www.mdpi.org

\title{
Synthesis of 2,4,6-Tri-substituted-1,3,5-Triazines
}

\author{
Carlos A. M. Afonso ${ }^{1, *}$, Nuno M. T. Lourenço ${ }^{2}$ and Andreia de A. Rosatella ${ }^{2}$ \\ 1 CQFM, Departamento de Engenharia Química, Instituto Superior Técnico, 1049-001 Lisboa, \\ Portugal; Fax + 351218464455 . \\ 2 REQUIMTE, Departamento de Química, Faculdade de Ciências e Tecnologia, Universidade Nova \\ de Lisboa, 2829-516 Caparica, Portugal.
}

* Author to whom correspondence should be addressed; e-mail: carlosafonso@ist.utl.pt

Received: 21 July 2005; in revised form: 8 September 2005 / Accepted: 8 September 2005 / Published: 31 January 2006

\begin{abstract}
Several specific synthetic protocols were developed for the preparation from cyanuric chloride of a range of symmetric and non-symmetric di- and tri-substituted 1,3,5-triazines containing alkyl, aromatic, hindered, chiral and achiral hydroxyalkyl, ester and imidazole groups via sequential nucleophilic substitution of the $\mathrm{C}-\mathrm{Cl}$ bond by $\mathrm{C}-\mathrm{O}$, C-N and C-S bonds.
\end{abstract}

Keywords: Cyanuric chloride, s-triazine, 1,3,5-triazine, melamine derivatives.

\section{Introduction}

1,3,5-Triazines (or s-triazines) are a class of compounds well known for a long time, and still continue the object of considerable interest, mainly due to their applications in different fields, including the production of herbicides and polymer photostabilisers [1]. Some 1,3,5-triazines display important biological properties (Figure 1); for example hexamethylmelamine (HMM, 1) and 2-amino4-morphlino-s-triazine (2) are used clinically due to their antitumor properties to treat lung breast and ovarian cancer, respectively [2]. Hydroxymethylpentamethylmelamine (HMPMM, 3) is also the hydroxylated metabolite which corresponds to the major active form of HMM [3]. More recently, significant aromatase inhibitory activity were observed for 1,3,5-triazines of general structure 4. For the similar general structure 5 antitumor activity in human cancer and murine leukemia cell lines were observed [3]. The 1,3,5-triazine 6 presents potential use as siderophore (microbial iron shelter) 
mediated drug [4] and the general structure 7 presents potent corticotrophin-releasing factor receptor antagonist activity [5]. The compounds of type 8 show potent activity against leukotriene $\mathrm{C}_{4}\left(\mathrm{LTC}_{4}\right)$ antagonist, which possess a protective effect on $\mathrm{HCl}$.ethanol-induced gastric lesions [6]. More recently it was discovered that the compound $\mathbf{9}$ is a potent corticotrophin-releasing factor ${ }_{1}$ receptor antagonist [7]. Among several other 1,3,5-triazine substituted polyamines tested, the substrate $\mathbf{1 0}$ presents a good in vitro activity against the protozoan parasite Trypanosoma brucei, the causative organism of Human African Trypanosomasis [8].

Figure 1. Select examples of biologically active compounds containing the 1,3,5-triazine unit.<smiles>CN(C)c1nc(N(C)C)nc(N(C)C)n1</smiles>

HMM

1

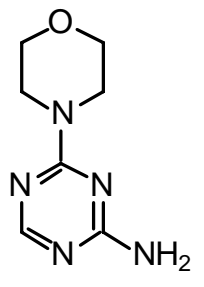

2<smiles>CN(C)c1nc(N(C)C)nc(N(C)CO)n1</smiles>

HMPMM

3<smiles>[R]c1nc([R])nc(N2CCOCC2)n1</smiles>

$\mathrm{R}^{1}, \mathrm{R}^{2}=$ morpholine, imidazole

4<smiles>[R]c1nc(N2CCOCC2)nc(-n2cnc3ccccc32)n1</smiles>

$\mathrm{R}=$ dimethylamine;

2,6-dimethyl-morpholine

5<smiles>[R]N(CC1CC1)c1nc(C)nc(Nc2c(C)cc(C)cc2C)n1</smiles>

7<smiles>COCC(COC)Nc1nc(C)nc2c(-c3ccc(Cl)cc3Cl)c(C)nn12</smiles>

9<smiles>[R]c1nc([R])nc([R])n1</smiles><smiles>[R]=NCOCCCCN(O)C(C)=O</smiles>

6<smiles>[R]C=Cc1nc(N)nc(N)n1</smiles><smiles>[R]=Cc1cccnc1</smiles>

8<smiles>CNc1nc(CC(Cc2nc(NC)nc(NC)n2)NCCCNC(C)NCCCN(Cc2nc(NC)nc(NC)n2)Cc2nc(NC)nc(NC)n2)nc(NC)n1</smiles>

10

The diverse biological activities observed for different molecules containing the 1,3,5-triazine unit have been further explored in order to discover other new potential molecules through the synthesis of libraries by combinatorial approaches [9]. The 1,3,5-triazine unit has also been used as a key functional group in host-guest chemistry, mainly based on the possibility to generate organized 
aggregates via the formation of strong three simultaneous hydrogen bonds 11 [10]. Other applications of the 1,3,5-triazine derivatives are: i) as chiral stationary phases, for example, the chiral solvating agent 12 for the determination of enantiomeric excess by NMR spectroscopy [11] and determination of absolute configuration by circular dichroism [12]; ii) for the preparation of luminescent, optical switches and tri-radical cation species in the case of 2,4,6-triamino-1,3,5-triazine compounds of general structure 13 [13]; iii) as metal complexes, liquid crystals, calixarenes, dendrimers, polymers and optical brighteners for household washing powders 14 [14].

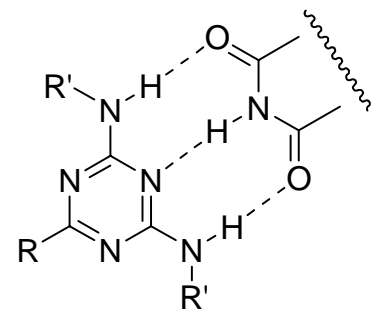

11<smiles>[R]c1nc([R])nc([R])n1</smiles>

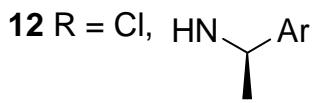

$13 \mathrm{R}=\mathrm{NAr}_{2}$
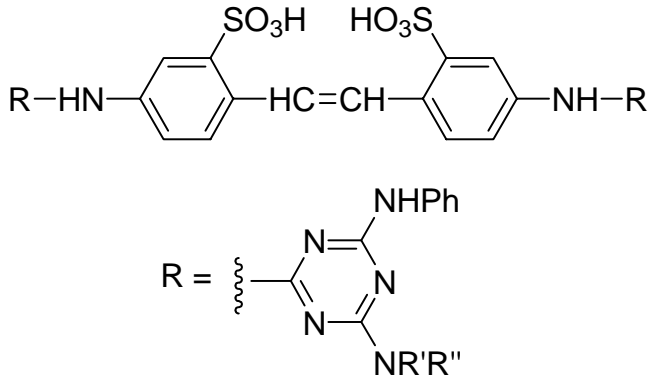

14

The most practical method for the synthesis of substituted 1,3,5-triazines is based on the functionalization of the less expensive reagent cyanuric chloride by successive, controlled nucleophilic substitution of each chloride, taking advantage of the decrease of reactivity with the number of substituents [15] (Scheme 1).

Scheme 1. Ease of chloride substitution on chlorinated 1,3,5-triazines by nucleophiles (Nu): cyanuric chloride $>\mathbf{1 5}>\mathbf{1 6}$.<smiles>Clc1nc(Cl)nc(Cl)n1</smiles>

cyanuric chloride<smiles>N#[W]c1nc(Cl)nc(Cl)n1</smiles>

15<smiles>N#[W]c1nc([Al])nc(Cl)n1</smiles>

16<smiles>N#[W]c1nc([AlH2])nc([AlH2])n1</smiles>

17

These reactivity profile has been explored in the synthesis of a large number of 1,3,5-triazines containing different substituents, using combinatorial synthesis [9] and for development of solid phase methodologies [16]. Cyanuric acid has also been used as a versatile reagent for the conversion of alcohols to formates and alkyl chlorides [17], carboxylic acids into 2-oxazolines, acid chlorides, esters, hydroxamic acids, amides [18] and alcohols [19], as an alternative method to classical Swern oxidation [20], $N$-dimethylation of tertiary amines and in the Beckman rearrangement of oximes [21]. The derivative 2-chloro-4,6-dimethoxy-1,3,5-triazine has also been applied for ester synthesis [22] and as an enantiodifferentiating coupling reagent [23]. Other applications of cyanuric chloride are found in agriculture, the textile industry and the dye chemistry [1,24]. In the case of 1,3,5-triazines containing 
electron-donating groups, such as the amino group in the positions 2,4 or 6 , a stronger bond is generated which causes more restriction to free rotation [24].

In the course of recent studies on the synthesis of 2,4,6-tri-substituted 1,3,5-triazines containing hindered amines [25], we also developed synthetic procedures which allow the preparation of a range of 1,3,5-triazines containing structurally different groups attached to the 1,3,5-triazine core, namely by substituents with potential coordinating groups. Here we report on the preparation of a series of new 2,4,6-tri-substituted-1,3,5-triazines via sequential substitution of the three chlorides of cyanuric chloride by O-, $\mathrm{N}$ - and S- centered nucleophiles.

\section{Results and Discussion}

The complete range of 2,4,6-trisubstituted-1,3,5-triazines 18-22 were obtained from cyanuric chloride by sequential substitution of the chloride atom using oxygen, nitrogen and sulfur centered nucleophiles a-y (Scheme 2).

The 2,4,6-trialkoxy-1,3,5-triazines 18, were prepared in moderate to high yields (52 - $89 \%$ ) by reaction of the corresponding lithium alkoxide with cyanuric chloride (method i, Scheme 3 ). The introduction of only two amino groups was easily achieved by performing the reaction at ambient temperature (method iii), taking advantage of a considerable decrease of reactivity with the number of substituents. This procedure allowed us to prepare the symmetric 2-chloro-4,6-diamino-1,3,5-triazines 19 (Scheme 3) in reasonable yields (44 - $98 \%$ ). Additionally, reacting the cyanuric chloride firstly with the alkoxide, followed by second addition of the amine group allows the preparation of 2-chloro4-alkoxy-6-amino-1,3,5-triazines 21 (Scheme 4, methods vi-x). In the case of the addition of the more hindered amines $\mathbf{q}$ and $\mathbf{r}$ were necessary to use higher temperature (method ix). The addition became even more difficult in the case of the amino group $\mathbf{q}$ in the precursor containing the hindered group $t$ butoxide $\mathbf{0}$. In this case, the disubstituted triazine $\mathbf{2 1 0 q}$ was only obtained in $10 \%$ by refluxing the reaction mixture in THF (Scheme 4, method $\mathrm{x}$ ). A similar approach was used for the preparation of the derivatives 23 containing two different donating groups (amines and thiols) by sequential addition of each amine to cyanuric chloride and control of the reaction temperature (Scheme 5, method vii). However, this approach was unsuccessful for the amine $\mathbf{m}$ which contains the free imidazole group.

The substitution of the remaining chloride atom by thio or amino groups required considerably more vigorous conditions. The experimental conditions were optimized for the substrate $\mathbf{1 9 f}_{2}$. Using the reported conditions $\left(\mathrm{H}_{2} \mathrm{O} / \mathrm{NH}_{4} \mathrm{OH} / 1\right.$,4-dioxane, $\left.120{ }^{\circ} \mathrm{C}\right)$ [26] or the conditions $\mathrm{PhSH} / \mathrm{i}-\mathrm{Pr}_{2} \mathrm{NEt} / \mathrm{THF}$, $120{ }^{\circ} \mathrm{C}$ no addition of the $\mathrm{NH}_{2}$ or $\mathrm{PhS}$ groups were observed respectively. However, in case of the following experimental conditions ( $\mathrm{PhSH} / \mathrm{AgOAc} / \mathrm{K}_{2} \mathrm{CO}_{3} / \mathrm{EtOH} / \mathrm{H}_{2} \mathrm{O}, 140{ }^{\circ} \mathrm{C}$ or $\mathrm{PhSH} / \mathrm{KOH} / 1,4-$ dioxane, reflux, method iv) the desired product $\mathbf{2 0 f}_{2} \mathbf{k}$ was obtained in $80 \%$ and $97 \%$ respectively

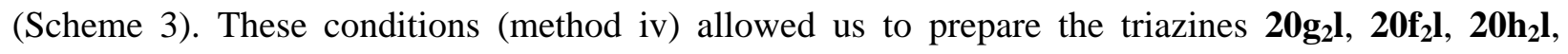
22nps, 22npk and 25pts in high yields (77 - $97 \%$ ). The substitution of $\mathrm{Cl}$ by the primary amines $\mathbf{m}$ and $\mathbf{y}$ on the substrate $\mathbf{1 9} \mathbf{g}_{2}$ and $\mathbf{1 9} \mathbf{x}_{\mathbf{2}}$ was achieved respectively in high (89 \%) to moderate (50 \%) yields by refluxing solution of 1,4-dioxane or 1,2-dichloroethane in the presence of an excess of the corresponding amine (method v). However, other attempts to react the substrate with more hindered amines as for example secondary amines under the above conditions (method v) were unsuccessful. 
Scheme 2. General synthetic pathway used for the synthesis of 2,4,6-trisubstituted-1,3,5triazines<smiles>[R20]Nc1nc([R20])nc(Cl)n1</smiles>

19<smiles>[R2]Nc1nc([R])nc(N[R20])n1</smiles>

20<smiles>Clc1nc(Cl)nc(Cl)n1</smiles>

cyanuric chloride

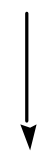<smiles>[R]Oc1nc([R20])nc([R])n1</smiles>

18<smiles>[R20]c1nc(Cl)nc(N([R2])[R])n1</smiles>

21<smiles>[Y][Y]c1nc([R20])nc([R6])n1</smiles>

22

Used Groups a-y: RO, $\mathrm{R}_{2} \mathrm{~N}, \mathrm{RNH}, \mathrm{RS}$<smiles>[R]N1CCC[C@H]1CO</smiles>

a $\mathrm{R}^{\prime}=\mathrm{Boc}$

b $\mathrm{R}^{\prime}=\mathrm{H}$<smiles>C[C@H]([C@H](O)c1ccccc1)N(C)Cc1ccccc1</smiles>

C<smiles>CNCCOCCNC[PbH2]c1ccn(CCS)c1</smiles><smiles>c1c[nH]cn1</smiles>

k

I<smiles>CN[C@@H](C)C(O)c1ccccc1</smiles><smiles>CN[C@@H](C)[C@H](O)c1ccccc1</smiles><smiles>OC(c1ccccc1)(c1ccccc1)C1CCCN1</smiles>

e

f

g

h

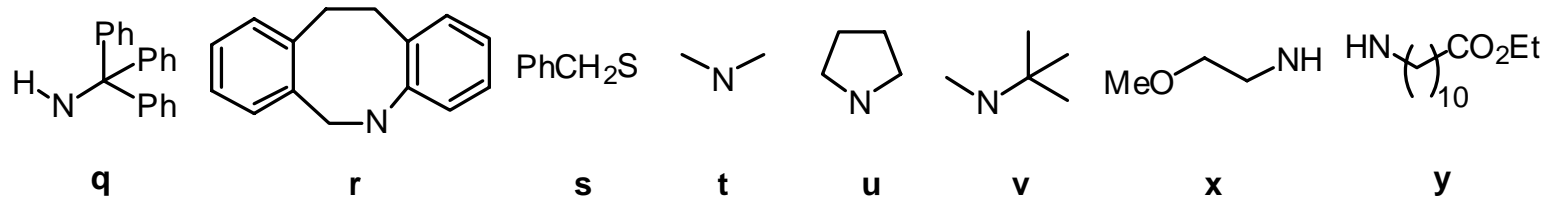


Scheme 3. Synthesis of 2,4,6-trisubstituted-1,3,5-triazines 18, 19 and 20 from cyanuric chloride.

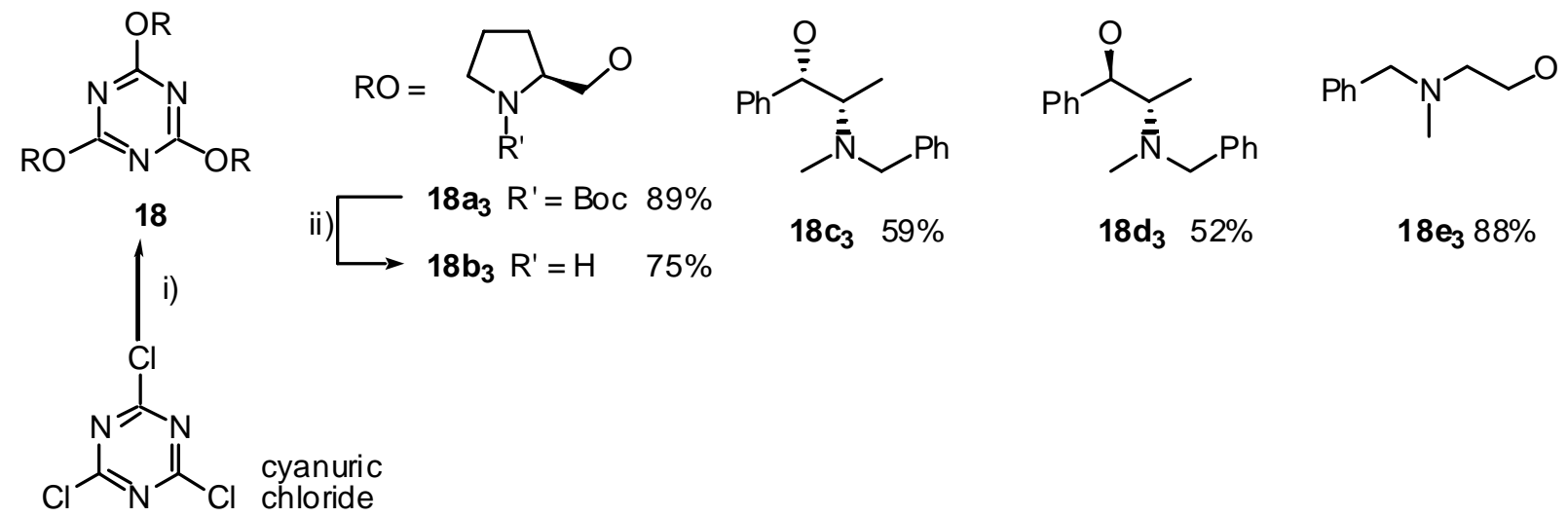<smiles></smiles><smiles>[R20]Nc1nc([R]#N)nc(N[R20])n1</smiles>

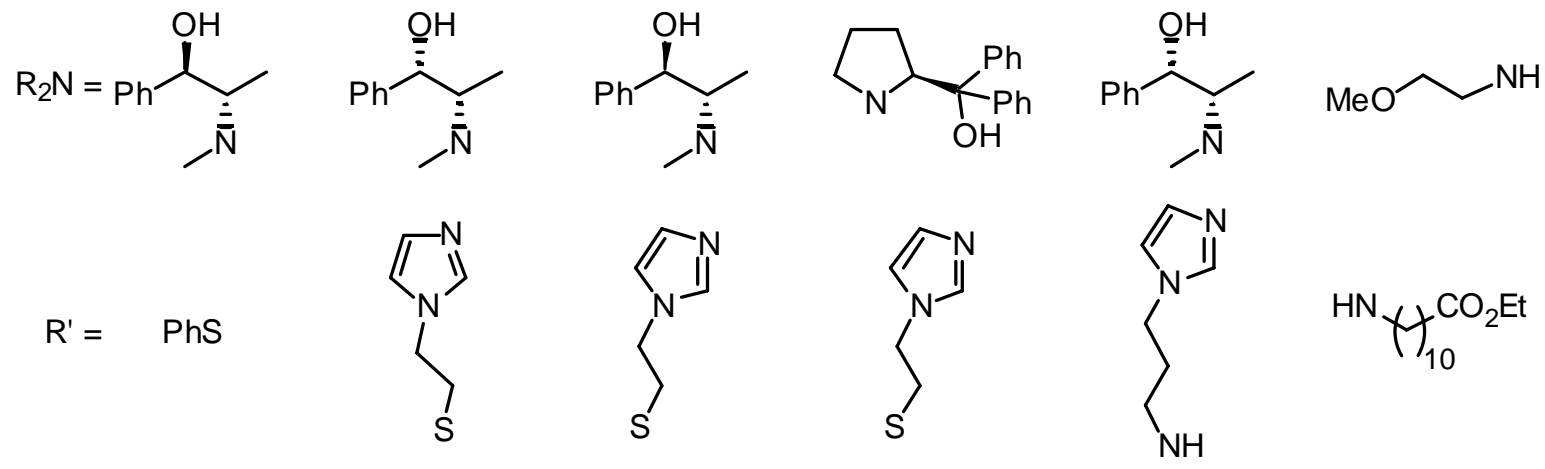

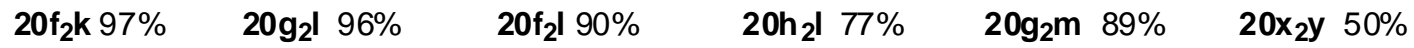

Methods: i) cyanuric chloride, ROLi (3 eq.), THF, $0{ }^{\circ} \mathrm{C}$ to rt; ii) $\mathrm{CF}_{3} \mathrm{CO}_{2} \mathrm{H}, 0^{\circ} \mathrm{C}$; iii) cyanuric chloride, diisopropylethylamine (2.2 eq.), $\mathrm{R}_{2} \mathrm{NH}$ (2.1 eq.), $\mathrm{CH}_{2} \mathrm{Cl}_{2}, 0{ }^{\circ} \mathrm{C}$ to rt; iv) 2-chloro-1,3,5triazine, thiol (2 eq.), KOH (2.2 eq.), 1,4-dioxane, reflux; v) $19 \mathbf{g}_{2}$, amine (5 eq.), 1,4-dioxane, reflux. 
Scheme 4. Synthesis of alkoxy-1,3,5-triazines 21 and 22 from cyanuric chloride.<smiles>Clc1nc(Cl)nc(Cl)n1</smiles>

cyanuric chloride
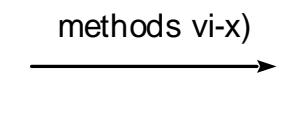

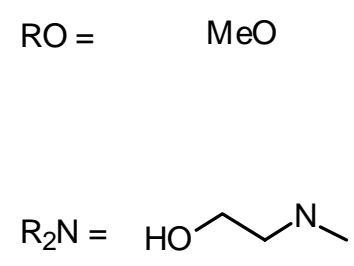<smiles>CCNC</smiles><smiles>CCNC</smiles><smiles>[Tl]C(c1ccccc1)(c1ccccc1)c1ccccc1</smiles><smiles>CC(C)(C)O</smiles>

$\mathrm{MeO}$

method vi)

21ni $28 \%^{\mathrm{a}}$ $+$

$24 n_{2}$ i $40 \%{ }^{a}$<smiles>COc1nc(OC)nc(N(C)CCO)n1</smiles>

$24 n_{2} \mathrm{i}$<smiles>[R2]Nc1nc([R20])nc(Cl)n1</smiles>

21
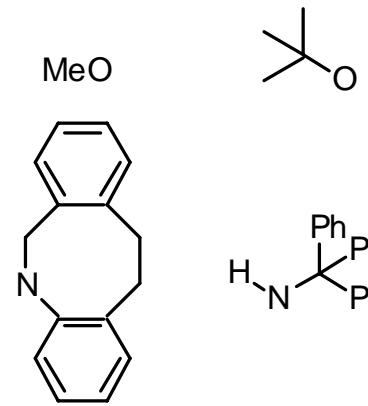<smiles>[Tl]C(c1ccccc1)(c1ccccc1)c1ccccc1</smiles>

method $x)$

$210 \mathrm{1} 10 \%$

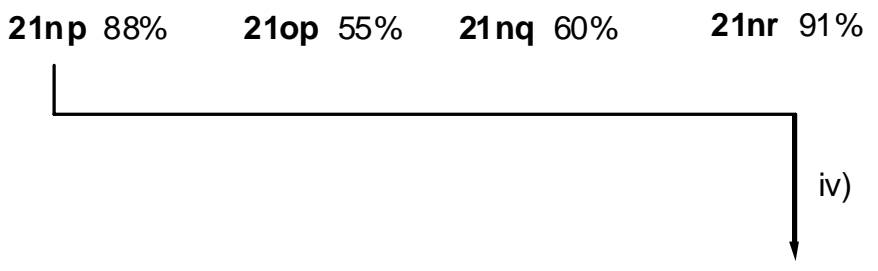<smiles>[R7]c1nc(OC)nc(N(C)CC)n1</smiles>

22

Methods: iv) 2-chloro-1,3,5-triazine, thiol (2 eq.), KOH (2.2 eq.), 1,4-dioxane, reflux; vi) MeONa (2 eq.), cyanuric chloride, THF, $0{ }^{\circ} \mathrm{C}$ to rt., then diisopropylethylamine (1.2 eq.), amine (1.1 eq.), rt; vii) cyanuric chloride, diisopropylethylamine (2.2 eq.), $\mathrm{MeOH}$ (1.0 eq.), $\mathrm{CH}_{2} \mathrm{Cl}_{2}, 0$ ${ }^{\circ} \mathrm{C}$ to $\mathrm{rt}$, then $\mathrm{R}_{2} \mathrm{NH}$ (1.1 eq.), rt; viii) cyanuric chloride, $t$-BuOLi (1 eq.), THF, $\mathrm{rt}$, then diisopropylethylamine (1.1 eq.), $\mathrm{R}_{2} \mathrm{NH}$ (1.0 eq.), rt; ix) cyanuric chloride, diisopropylethylamine (2.2 eq.), DMAP (cat.), $\mathrm{MeOH}$ (1.0 eq.), $\mathrm{CH}_{2} \mathrm{Cl}_{2}$, rt, then $\mathrm{R}_{2} \mathrm{NH}$ (1.1 eq.), reflux; $\mathrm{x}$ ) cyanuric chloride, $t$-BuOLi (1 eq.), THF, rt , then DMAP (cat.), diisopropylethylamine (1.1 eq.), $\mathrm{R}_{2} \mathrm{NH}$ (1.0 eq.), reflux; ${ }^{a}$ the triazine $\mathbf{2 4 n}_{\mathbf{2}} \mathbf{i}$ was also obtained in $40 \%$ yield. 
Scheme 5. Synthesis of 2,4,6-trisubstituted-1,3,5-triazines 23 from cyanuric chloride by sequential substitution of chlorides.<smiles>Clc1nc(Cl)nc(Cl)n1</smiles>

cyanuric chloride vii)

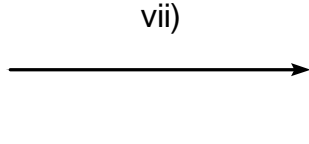

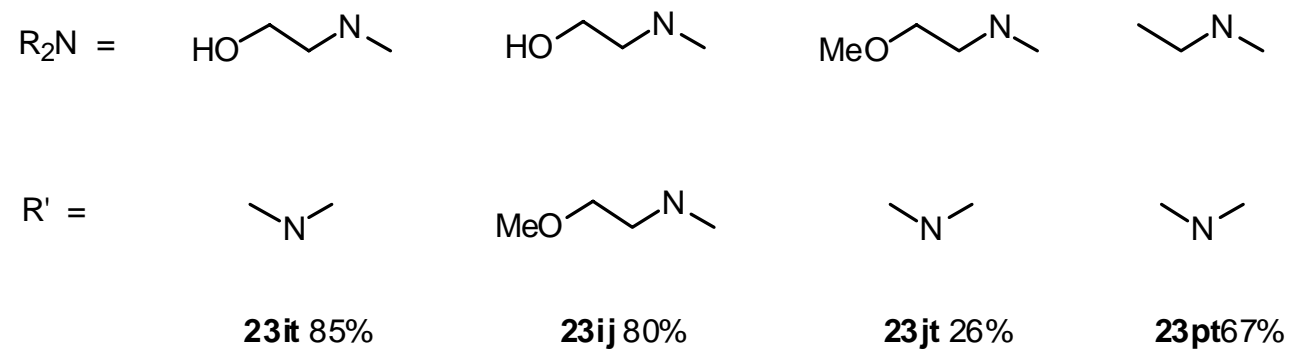

$\mathrm{R}_{2} \mathrm{~N}=$<smiles>CCNC</smiles><smiles>CCNC</smiles><smiles>[N]C(c1ccccc1)(c1ccccc1)c1ccccc1</smiles><smiles>C1CCNC1</smiles>

23pu $72 \%$

$\mathrm{R}^{\prime}=$<smiles>SC[Pb]</smiles>

23ps $72 \%$<smiles>CNC(C)(C)C</smiles>

$\mathrm{HN}_{Y} \mathrm{CO}_{10}^{\mathrm{CO}_{2} \mathrm{Et}}$

23qv 54\%

$23 x y 69 \%$<smiles>[R]c1nc(Cl)nc(N([R])[X])n1</smiles>

23<smiles>CCN(C)c1nc(Cl)nc(N(C)C)n1</smiles>

$23 \mathrm{pt}$ iv)<smiles>CCN(C)c1nc(C)nc(N(C)C)n1</smiles>

25pts $68 \%$

Methods: iv) 1,3,5-triazine 23pt, thiol (2 eq.), $\mathrm{KOH}$ (2.2 eq.), 1,4-dioxane, reflux; vii) cyanuric chloride, diisopropylethylamine (2.2 eq.), $\mathrm{R}_{2} \mathrm{NH}$ (1.0 eq.), $\mathrm{CH}_{2} \mathrm{Cl}_{2},{ }^{\circ}{ }^{\circ} \mathrm{C}$ to rt, then $\mathrm{R}$ 'H (1.1 eq.), rt. 


\section{Conclusions}

The synthetic procedures described here allows the synthesis of a range of symmetric and nonsymmetric di- and tri-substituted-1,3,5-triazines containing a range of substituents attach to the triazine core containing asymmetric centers and functional groups such as alkyl, aromatic, including more hindered ones, chiral and non-chiral hydroxyalkyl, ester and imidazole groups.

\section{Acknowledgments}

We would like to thank Fundação para a Ciência e Tecnologia and FEDER for financial support (Ref. POCTI/QUI/60175/2004 and SFRH/BD/18487/2004) and Prof. W. B. Motherwell for helpful comments and for some laboratory facilities.

\section{Experimental}

\section{General}

Reagent quality solvents were distilled prior to use. Triethylamine, diisopropylethylamine and dichloromethane $\left(\mathrm{CH}_{2} \mathrm{Cl}_{2}\right)$ were distilled from $\mathrm{CaH}_{2}$ (in the case of the amines) and $\mathrm{P}_{2} \mathrm{O}_{5}$, respectively. Anhydrous benzene, toluene, tetrahydrofuran (THF) and diethyl ether $\left(\mathrm{Et}_{2} \mathrm{O}\right)$ were prepared by distillation from sodium/benzophenone ketyl under argon. Column chromatography was performed using Silica gel $60 \mathrm{MN}$ and aluminium-backed silica gel Merck $60 \mathrm{~F}_{254}$ plates was used for analytical TLC. Melting points (uncorrected) were determined on a Electrothermal Mod. IA 6304 capillary melting point apparatus. Microanalyses were carried out at ITQB (Portugal) using a Carlo Erba analyser. Mass spectra (MS) and accurate masses (HRMS) were obtained from the Mass Spectrometry Services, School of Pharmacy, University of London and University of Santiago de Compostela (Spain). Infrared spectra (IR) were recorded on a Buck Scientific Mod. 500 and Jasco FT/IR-430 spectrophotometers. ${ }^{1} \mathrm{H}$ - and ${ }^{13} \mathrm{C}-\mathrm{NMR}$ were recorded on a Bruker ARX 400 spectrometer. Chemical shifts are reported as $\delta$ values relative to tetramethylsilane $\left(\delta_{\mathrm{H}}=0 \mathrm{ppm}\right), \mathrm{CDCl}_{3}\left(\delta_{\mathrm{C}}=77.0\right)$. Due to the occurrence of hindered rotation for some triazines [24], for some examples the NMR were performed at higher temperatures. All coupling constants are given in Hz. Observed rotations at the Na-D line were measured at $25^{\circ} \mathrm{C}$ using a Optical Activity polarimeter Mod. AA-1000.

Typical procedures: Method i): $n$-Butyllithium (1.2 mL, $1.0 \mathrm{M}, 1.2 \mathrm{mmol}$ ) was added dropwise (3 min.) to a stirred solution of (S)- $N$-tert-butoxycarbonyl prolinol [27] (237.8 $\mathrm{mg}, 1.18 \mathrm{mmol}$ ) in anhydrous THF $(11 \mathrm{~mL})$ at $0{ }^{\circ} \mathrm{C}$ under argon. After $3 \mathrm{~min}$. a solution of cyanuric chloride (70.5 mg, $0.38 \mathrm{mmol}$ ) in anhydrous THF (2 $\mathrm{mL})$ was added dropwise via cannula $(4 \mathrm{~min}$.) and the reaction mixture was then allowed to warm to room temperature. After $14 \mathrm{hr}$ the mixture was partitioned between diethyl ether (30 mL) and saturated aqueous ammonium chloride (30 mL). The aqueous phase was extracted with $\mathrm{Et}_{2} \mathrm{O}(2 \times 30 \mathrm{~mL})$, the combined organic layers were dried $\left(\mathrm{MgSO}_{4}\right)$, filtered, evaporated to dryness and purified by flash chromatography (7:3 hexane/Et $\left.\mathrm{E}_{2} \mathrm{O}\right)$ to afford $\mathbf{1 8 \mathbf { a } _ { 3 }}(229.7$ mg, $89 \%$ ) as a white solid; $[\alpha]^{25} \mathrm{D}=-93.10$ (c 1.1, $\left.\mathrm{CHCl}_{3}\right) ; v_{\max }(\mathrm{film}) / \mathrm{cm}^{-1}$ 2974, 2928, 2883, 1696 
(CO), 1566, 1401, 1323, 1253, 1168, 1134, 907, 816, 770. 736; $\delta_{\mathrm{H}}$ (400 MHz; $\left.\mathrm{CDCl}_{3}\right) 1.45$ (9H, s t-Bu), 1.9-2.0 (4H, m), $3.39(2 \mathrm{H}$, br, $\left.\mathrm{CH}_{2} \mathrm{~N}\right)$, 4.1-4.6 (3H, m, $\mathrm{CHN}$ and $\left.\mathrm{CH}_{2} \mathrm{O}\right) ; \delta_{\mathrm{C}}\left(\mathrm{CDCl}_{3}\right) 22.79$, 23.64, 27.95, 28.42, 28.68, 46.37, 46.75, 55.46, 67.98, 68.19, 79.36, 79.65, 154.4, 173; m/z (FAB) $679\left(\mathrm{M}^{+}\right)$, 579, 496, 396, 313, 239; HRMS calcd. for $\mathrm{C}_{33} \mathrm{H}_{55} \mathrm{~N}_{6} \mathrm{O}_{9}$ : 679.4031, found: 679.4035 .

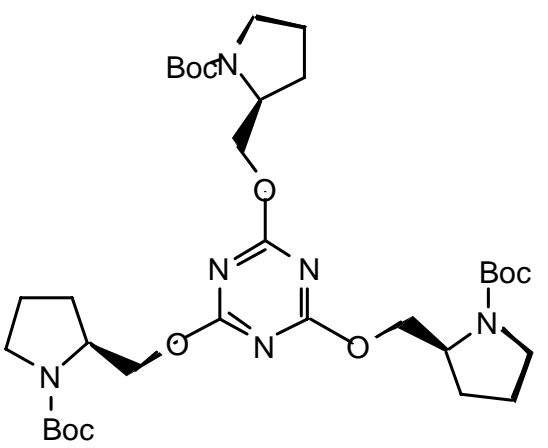

18c $c_{3}$ : Purified by flash chromatography (hexane/Et $\mathrm{t}_{2} \mathrm{O} 1: 1$ ); white spongy solid; $[\alpha]^{25} \mathrm{D}=+94.5$ (c $\left.0.7, \mathrm{CHCl}_{3}\right) ; v_{\max }$ (film) $/ \mathrm{cm}^{-1}$ 3088, 3071, 3037, 2974, 2940, 2883, 2855, 2804, 1571, 1497, 1458, $1400,1350,1327,1140,912,822,736,702 ; \delta_{\mathrm{H}}\left(400 \mathrm{MHz} ; \mathrm{CDCl}_{3}\right)$ 0.82 (3H, d J 6.8, 3-H), 2.06 (3H, s, NMe), 3.26 (1H, dt J 8.8 and 6.8, 2-H), 3.52 (1H, d J 14, $\mathrm{CH}_{2} \mathrm{Ph}$ ), 3.60 (1H, d J 14, $\left.\mathrm{CH}_{2} \mathrm{Ph}\right), 5.98$ (1H, d J 8.8, 1-H), 7.1-7.5 (10H, m, Ph); $\delta_{\mathrm{C}}\left(\mathrm{CDCl}_{3}\right)$ 11.6, 36.6, 58.7, 62.0, 80.9, 126.3, 127.5, 127.8, 128.0, 128.1, 128.3, 139.1, 139.9, 172.5; m/z (FAB) $842\left(\mathrm{MH}_{2}^{+}\right), 694,661,238,148$; HRMS calcd. for $\mathrm{C}_{54} \mathrm{H}_{61} \mathrm{~N}_{6} \mathrm{O}_{3}$ : 841.4805, found: 841.4808.

18d $_{3}$ : Purified by flash chromatography (hexane/Et ${ }_{2} \mathrm{O} 6: 4$ ); white solid; $v_{\max }(\mathrm{film}) / \mathrm{cm}^{-1}$ 3093, 3065, 3031, 2985, 2951, 2889, 2855, 2804, 1571, 1554, 1497, 1452, 1401, 1344, 1134, 912, 822, 736, 702; $\delta_{\mathrm{H}}\left(400 \mathrm{MHz} ; \mathrm{CDCl}_{3}\right) 1.08(3 \mathrm{H}, \mathrm{d} \mathrm{J}$ 6.8, 3-H), 2.12 (3H, s, $\mathrm{NMe}), 3.10$ (1H, quint. J 6.8, 2-H), $3.45\left(1 \mathrm{H}, \mathrm{d} \mathrm{J} 12.6, \mathrm{CH}_{2} \mathrm{Ph}\right)$, $3.50\left(1 \mathrm{H}, \mathrm{d} \mathrm{J} 12.6, \mathrm{CH}_{2} \mathrm{Ph}\right), 5.92(1 \mathrm{H}, \mathrm{d} \mathrm{J} 6.4,1-\mathrm{H}), 6.97-7.29$ $(10 \mathrm{H}, \mathrm{m}, \mathrm{Ph}) ; \delta_{\mathrm{C}}\left(\mathrm{CDCl}_{3}\right)$ 9.47, 37.41, 58.45, 62.03, 80.3, 126.6, 126.7, 127.4, 128.0, 128.4, 139.6, 140.0, 172.5; m/z (FAB) 841 $\left(\mathrm{MH}^{+}\right)$, 254, 238, 148, 91; HRMS calcd. for $\mathrm{C}_{54} \mathrm{H}_{61} \mathrm{~N}_{6} \mathrm{O}_{3}$ : 841.4805, found: 841.4803 .
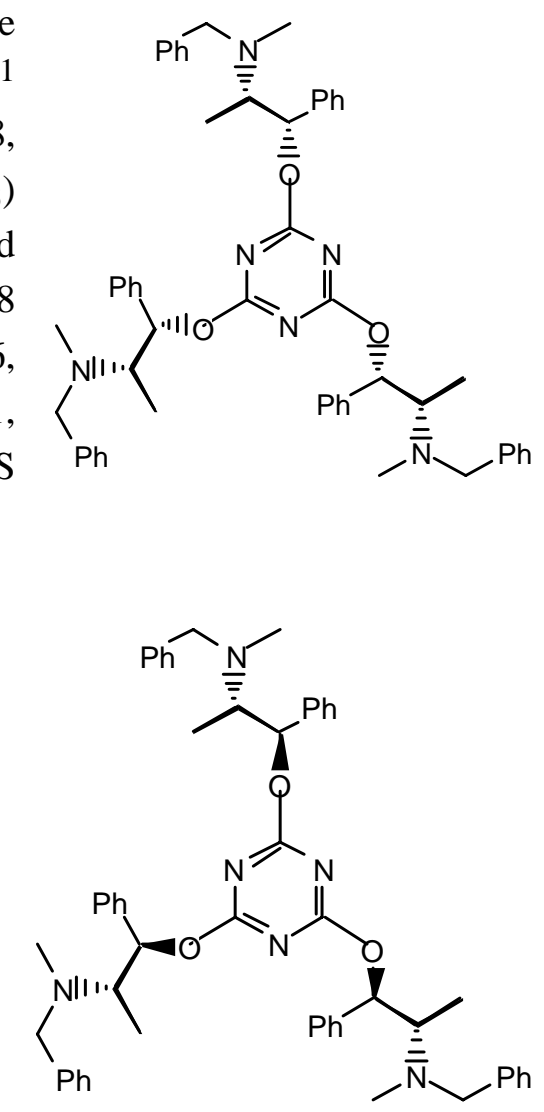

$\mathbf{1 8 e}_{3}$ [33]: Purified by flash chromatography (ethyl acetate/ethanol 8:2); colorless viscous liquid; $v_{\max }$ (film)/cm-1 3076, 3077, 2963, 2849, 2804, 1571, 1458, 1418, 1384, 1338, 1247, 1154, 1049, 1032, 822, 736, 702; $\delta_{\mathrm{H}}\left(400 \mathrm{MHz} ; \mathrm{CDCl}_{3}\right) 2.30(3 \mathrm{H}, \mathrm{s}, \mathrm{NMe})$, 2.81 (2H, t J 5.9, 2-H), 3.58 (2H, s, $\left.\mathrm{CH}_{2} \mathrm{Ph}\right), 4.48$ (2H, t J 5.9, 1-H), 7.21-7.29 (5H, m, Ph); $\delta_{\mathrm{C}}\left(\mathrm{CDCl}_{3}\right)$ 42.5, 54.9, 62.3, 65.9, 126.8, 128.0, 128.71, 138.5, 172.6; $\mathrm{m} / \mathrm{z}$ (FAB) $571\left(\mathrm{MH}^{+}\right), 477,422,299,285,275$; HRMS calcd. for $\mathrm{C}_{33} \mathrm{H}_{43} \mathrm{~N}_{6} \mathrm{O}_{3}$ : 571.3397, found: 571.3390 .

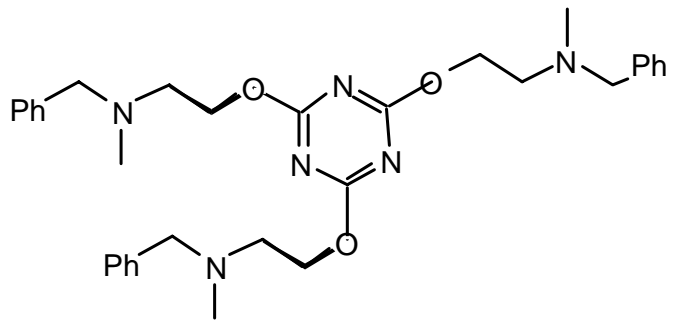

Method ii): To the triazine $\mathbf{1 8 a _ { 3 }}$ (229.7 $\mathrm{mg}, 0.34 \mathrm{mmol}$ ) at $0{ }^{\circ} \mathrm{C}$ was added trifluoracetic acid (2 ml) and the mixture was stirred for $20 \mathrm{~min}$. The acid was evaporated under vacuum and the residue was dissolved in $\mathrm{CH}_{2} \mathrm{Cl}_{2}(40 \mathrm{~mL})$ and was washed with saturated aqueous sodium bicarbonate $(25 \mathrm{~mL})$. 
The aqueous phase was extracted with $\mathrm{CH}_{2} \mathrm{Cl}_{2}(3 \times 20 \mathrm{~mL})$, the combined organic layers were dried $\left(\mathrm{MgSO}_{4}\right)$, filtered, evaporated to dryness and purified by flash chromatography on neutral alumina (ethanol) to afford $\mathbf{1 8 b}_{\mathbf{3}}$ (95.6 mg, $75 \%$ ) as a white solid; $[\alpha]^{25} \mathrm{D}=-114.2$ (c 2.5, $\mathrm{CHCl}_{3}$ ); $v_{\max }$ (film)/cm-1 3390 (NH), 2955, 2875, 1568, 1531, 1455, 1345, 1045, 808; $\delta_{\mathrm{H}}\left(400 \mathrm{MHz} ; \mathrm{CDCl}_{3}\right)$ 1.5-2.0 (12H, m), 2.9-3.0 (2H, m), 3.4$3.8(10 \mathrm{H}, \mathrm{m}), 4.2-4.3(6 \mathrm{H}, \mathrm{m}) ; \mathrm{m} / \mathrm{z}(\mathrm{FAB}) 378\left(\mathrm{M}^{+}\right), 348,322,296$, 263, 228, 198; HRMS calcd. for $\mathrm{C}_{18} \mathrm{H}_{31} \mathrm{~N}_{6} \mathrm{O}_{3}$ : 379.2458, found: 379.2453.

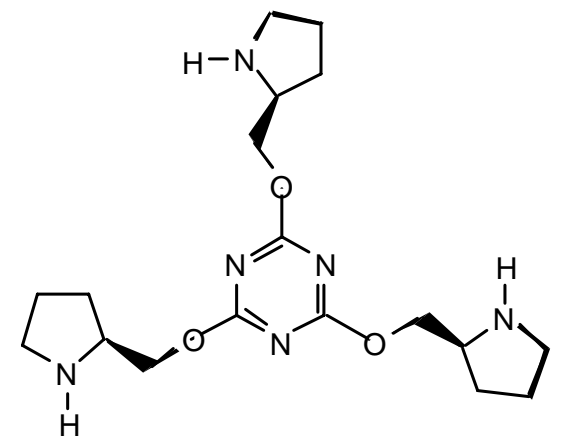

Method iii): To a stirred solution of (1S,2S)-pseudoephedrine (3.003 g, $18.17 \mathrm{mmol}$ ) and diisopropylethylamine (3.32 mL, $19.04 \mathrm{mmol})$ in anhydrous dichloromethane $(80 \mathrm{~mL})$ at $0{ }^{\circ} \mathrm{C}$ under argon, was added via cannula (6 min.) a solution of cyanuric chloride (1.595 g, $8.65 \mathrm{mmol}$ ) in $\mathrm{CH}_{2} \mathrm{Cl}_{2}$ (32 $\mathrm{mL}$ ) and the reaction mixture was allowed to warm to room temperature. After $14 \mathrm{hr}$ the mixture was partitioned between $\mathrm{CH}_{2} \mathrm{Cl}_{2}(50 \mathrm{~mL})$ and saturated aqueous sodium chloride $(100 \mathrm{~mL})$. The aqueous phase was extracted with $\mathrm{CH}_{2} \mathrm{Cl}_{2}(70 \mathrm{~mL})$, the combined organic layers were dried $\left(\mathrm{MgSO}_{4}\right)$, filtered, evaporated to dryness and purified by flash chromatography (hexane/ethyl acetate 7:3) to afford $\mathbf{1 9 g}_{2}$ (3.67 g, $96 \%$ ) as a white spongy solid; $[\alpha]^{25} \mathrm{D}=+182.2$ (c 1.0, $\mathrm{CHCl}_{3}$ ); $v_{\max }$ (film)/(cm ${ }^{-1}$ 3428 (OH), 3099, 3076, 3048, 2997, 2951, 2895, 1571, 1492, 1406, 1304, 1202, 1151, 1049, 975, 799, 759, 702; $\delta_{\mathrm{H}}\left(400 \mathrm{MHz}\right.$; DMSO-d $\left.6100{ }^{\circ} \mathrm{C}\right) 0.99(3 \mathrm{H}, \mathrm{d}$ J 5.6, 3H), 2.99 (3H, s, NMe), 4.68 (1H, br s), 4.89 (1H, br s), 5.02 (1H, br s), 7.2-7.4 (5H, m, Ar); $\delta_{\mathrm{C}}\left(\mathrm{DMSO}_{6}, \mathrm{~d}_{6}, 100{ }^{\circ} \mathrm{C}\right)$ 13.5, 28.7, 55.2, 73.8, 126.3, 126.6, 127.3, 142.9, 164.4; $\mathrm{m} / \mathrm{z}$ (FAB) $442\left(\mathrm{MH}^{+}\right)$, 424, 334, 316, 290, 228, 200; HRMS calcd. for $\mathrm{C}_{23} \mathrm{H}_{29} \mathrm{~N}_{5} \mathrm{O}_{2} \mathrm{Cl}$ : 442.2010, found: 442.2015 .

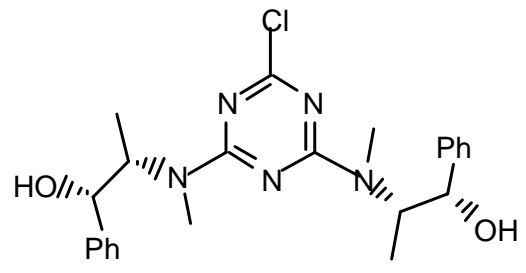

19f : $_{2}$ Purified by flash chromatography (hexane/ethyl acetate 7:3); white spongy solid; $[\alpha]^{25} \mathrm{D}=-150.1$ (c 1.0, $\left.\mathrm{CHCl}_{3}\right) ; v_{\max }$ (film)/cm $3394(\mathrm{OH}), 3088,3064,3031$, 2985, 2940, 2877, 1548, 1463, 1384, 1293, 1191, 1032, 963, 782; $\delta_{\mathrm{H}}\left(400 \mathrm{MHz}\right.$; toluene- $\left.\mathrm{d}_{8}, 95{ }^{\circ} \mathrm{C}\right) 1.07$ (3H, d J 7.0, CHMe), 2.84 (3H, s, NMe), 4.62 (1H, br s), 4.75 (1H, br s), 7.01-7.12 (3H, m, $\mathrm{Ph}), 7.27$ (2H, d J 6.8, Ph); $\mathrm{m} / \mathrm{z}$ (FAB) $442\left(\mathrm{MH}^{+}\right)$, 424, 406, 379,<smiles>C[C@H]([C@H](O)c1ccccc1)N(C)c1nc(Cl)nc(N(C)[C@H](O)c2ccccc2)n1</smiles>
334, 316; HRMS calcd. for $\mathrm{C}_{23} \mathrm{H}_{29} \mathrm{~N}_{5} \mathrm{O}_{2} \mathrm{Cl}$ : 442.2010, found: 442.2013.

19h 2 : Purified by flash chromatography (hexane/ $\mathrm{Et}_{2} \mathrm{O}$ 6:4); needles; m.p. 138-140 o $\mathrm{C}(\mathrm{EtOH}) ;[\alpha]^{25} \mathrm{D}=-258.6$ (c 1.1, $\left.\mathrm{CHCl}_{3}\right) ; v_{\max }$ (film)/cm-1 3411 (OH), 3095, 3062, 2985, 2891, 1556, 1490, 1344, 1299, 1147, 1020, 800, 762, 700; $\delta_{\mathrm{H}}\left(400 \mathrm{MHz}\right.$; toluene-d8, $95{ }^{\circ} \mathrm{C}$ ) 0.94 (1H, br s), 1.19 (1H, br s), $1.71(1 \mathrm{H}, \mathrm{m}), 1.80(1 \mathrm{H}$, br s), 3.08 (1H, br s), $3.64(1 \mathrm{H}$, dd J 8.0, 18.0), $5.06(1 \mathrm{H}, \mathrm{br} \mathrm{s}), 7.02-7.12(6 \mathrm{H}$, m), 7.34-7.45 (4H, m); m/z (FAB) $618\left(\mathrm{MH}^{+}\right), 600,582,522,434$,

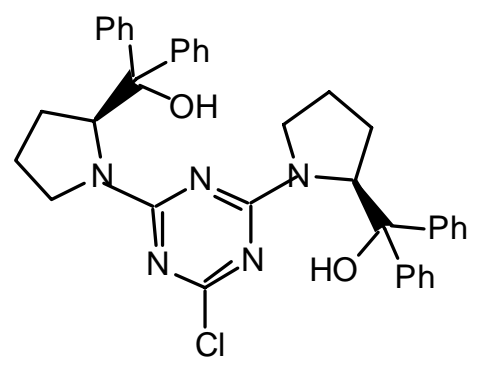


416; HRMS calcd. for $\mathrm{C}_{37} \mathrm{H}_{37} \mathrm{~N}_{5} \mathrm{O}_{2} \mathrm{Cl}$ : 618.2636, found: 618.2636.

19i $\mathbf{i}_{2}$ : Purified by flash chromatography (ethyl acetate); colorless viscous liquid; $v_{\max }(\mathrm{film}) / \mathrm{cm}^{-1} 3368(\mathrm{OH}), 2936,2875,1568$, 1520, 1489, 1403, 1310, 1204, 1043, 999, 970, 860, 798; $\delta_{\mathrm{H}}(400$ MHz; toluene-d8, $\left.95{ }^{\circ} \mathrm{C}\right) 2.83$ (3H, s, NMe), 3.33 (2H, t J 4.7), 3.45 (2H, t J 4.7); m/z (FAB) $262\left(\mathrm{MH}^{+}\right), 244,230,218,180,154$;<smiles>CN(CCO)c1nc(Cl)nc(N(C)CCO)n1</smiles>
HRMS calcd. for $\mathrm{C}_{9} \mathrm{H}_{17} \mathrm{~N}_{5} \mathrm{O}_{2} \mathrm{Cl}$ : 262.1071, found: 262.1080 .

19j2: Purified by flash chromatography ( $\mathrm{Et}_{2} \mathrm{O} /$ hexane 1:1); white cubes; m.p. 38-39 ${ }^{\circ} \mathrm{C}$ (hexane); $v_{\max }$ (film)/cm-1 2985, 2940, 2895, 2832, 1571, 1492, 1406, 1316, 1202, 1117, 1054, 997, 969, 799; $\delta_{\mathrm{H}}$ (400 MHz; toluene- $\left.\mathrm{d}_{8}, 95^{\circ} \mathrm{C}\right) 2.95$ (3H, s, NMe), 3.07 (3H, s, OMe), 3.32 (2H, t J 5.5), 3.52 (2H, t J 5.5); m/z (FAB) $289\left(\mathrm{M}^{+}\right)$, 237, 219, 205, 197, 180; m/z (FAB) $290\left(\mathrm{MH}^{+}\right), 258,244,231$, 214, 200; HRMS calcd. for $\mathrm{C}_{11} \mathrm{H}_{21} \mathrm{~N}_{5} \mathrm{O}_{2} \mathrm{Cl}$ : 290.1384, found: 290.1370; Anal. calcd. for $\mathrm{C}_{11} \mathrm{H}_{20} \mathrm{~N}_{5} \mathrm{O}_{2} \mathrm{Cl}$ : H 6.96 C $45.6 \mathrm{~N} 24.17$ \%. Found: H 6.71 C 45.85 N $24.12 \%$.

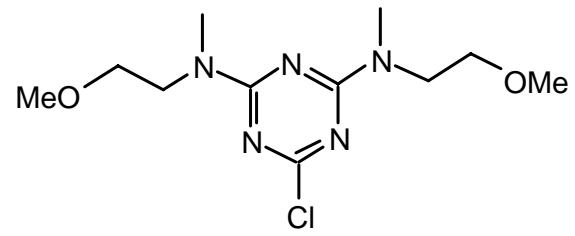

19x $x_{2}$ [28]: Recrystallized from ethyl acetate/hexane; white solid m.p 151-153 ${ }^{\circ} \mathrm{C} ; v_{\max }(\mathrm{NaCl}) / \mathrm{cm}^{-1}$; 3248, 3108, 2954, 2877, 1635, 1558, 1411, 1119, 794; $\delta_{\mathrm{H}}$ (400MHz; $\left.\mathrm{CDCl}_{3}\right), 3.34(3 \mathrm{H}, \mathrm{s} \mathrm{OMe}), 3.51(2 \mathrm{H}, \mathrm{d}, \mathrm{J}=4$ $\mathrm{CH}_{2} \mathrm{CH}_{2} \mathrm{OMe}$ ), $3.59\left(2 \mathrm{H}, \mathrm{d}, \mathrm{J}=4 \mathrm{CH}_{2} \mathrm{CH}_{2} \mathrm{OMe}\right) ; \delta_{\mathrm{C}}\left(\mathrm{CDCl}_{3}\right)$ 40.60, 58.81, 70.67, 165,54; m/z (CI) 262, $\left(\mathrm{MH}^{+}\right)$; HRMS calcd. for $\mathrm{C}_{9} \mathrm{H}_{17} \mathrm{ClN}_{5} \mathrm{O}_{2}$ : 262.107, found: 262.106.

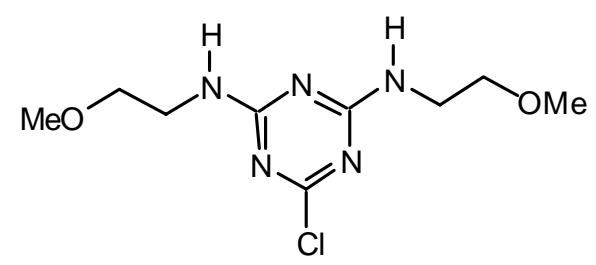

Method iv): A stirred mixture of 2-imidazol-1-yl-ethanethiol (l) [29] (204.4 mg, $0.92 \mathrm{mmol}$; contaminated with imidazole (43 \%)), triazine $\mathbf{1 9 g}_{2}(202.6 \mathrm{mg}, 0.46 \mathrm{mmol}$ ) and $\mathrm{KOH}$ (56.6 mg, 1.00 $\mathrm{mmol}$ ) in 1,4-dioxane $(1.5 \mathrm{~mL}$ ) under argon was refluxed during $1 \mathrm{hr}$ (formation of white precipitate). The mixture was partitioned between $\mathrm{CH}_{2} \mathrm{Cl}_{2}(20 \mathrm{~mL})$ and saturated aqueous ammonium chloride (25 $\mathrm{ml})$. The aqueous phase was extracted with $\mathrm{CH}_{2} \mathrm{Cl}_{2}(2 \times 20 \mathrm{~mL})$, the combined organic layers were dried $\left(\mathrm{MgSO}_{4}\right)$, filtered, evaporated to dryness and purified by flash chromatography $\left(\mathrm{Et}_{2} \mathrm{O} / \mathrm{ethanol}\right.$ 9.5:0.5) to afford $\mathbf{2 0 g}_{2} \mathbf{l}$ (233.9 $\mathrm{mg}, 96 \%$ ) as a white spongy solid; $[\alpha]^{25} \mathrm{D}=+140.7$ (c 1.0, $\mathrm{CHCl}_{3}$ ); $v_{\max }(\mathrm{film}) / \mathrm{cm}^{-1} 3338(\mathrm{OH}), 3190(\mathrm{OH}), 3116,3065,3037,2985,2940,2877,1548,1497,1401$, 1310, 1265, 1208, 1077, 1049, 1026, 975, 918, 804, 736, 702; $\delta_{\mathrm{H}}\left(400 \mathrm{MHz} ; \mathrm{CDCl}_{3}, 50{ }^{\circ} \mathrm{C}\right) 1.08(6 \mathrm{H}$, d J 6.9, CHMe), 3.07 (6H, s, NMe), 3.41 (2H, dq J 8.4 and 6.9, CHMe), 4.29 (2H, t J 7.1), 4.69 (2H, d J 8.4), 4.93 (2H, br s), 6.95 $(1 \mathrm{H}, \mathrm{s}), 7.03(1 \mathrm{H}, \mathrm{s}), 7.29-7.35(10 \mathrm{H}, \mathrm{m}, \mathrm{Ph}), 7.50(1 \mathrm{H}, \mathrm{s}) ; \delta_{\mathrm{C}}$ $\left(\mathrm{CDCl}_{3}, 50{ }^{\circ} \mathrm{C}\right)$ 14.4, 29.5, 30.5, 46.7, 56.8, 76.2, 118.7, 126.7, 126.8, 127.6, 128.2, 129.0, 136.9, 142.4, 164.2; m/z (FAB) 534 $\left(\mathrm{MH}^{+}\right)$, 516, 486, 466, 448, 426; HRMS calcd. for $\mathrm{C}_{28} \mathrm{H}_{36} \mathrm{~N}_{7} \mathrm{O}_{2} \mathrm{~S}$ : 534.2651 found: 534.2650 .

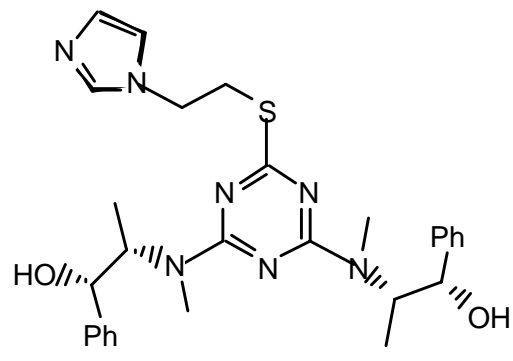


20f ${ }_{2}$ k: Purified by flash chromatography (hexane/ $\mathrm{Et}_{2} \mathrm{O}$ 6:4); white solid; $[\alpha]^{25} \mathrm{D}=-156.5$ (c 0.9, $\left.\mathrm{CHCl}_{3}\right) ; v_{\max }(\mathrm{film}) / \mathrm{cm}^{-1} 3401(\mathrm{OH}), 2927,1542,1492,1399$, 1304, 1264, 1208, 1175, 1050, 980, 803, 738, 702; $\delta_{\mathrm{H}}(400 \mathrm{MHz}$; toluene- $\left.\mathrm{d}_{8}, 95{ }^{\circ} \mathrm{C}\right) 1.03\left(6 \mathrm{H}, \mathrm{d} \mathrm{J}\right.$ 6.0, $\left.\mathrm{CHCH}_{3}\right), 2.34(2 \mathrm{H}$, br s), 2.78 (6H, s, NMe), 4.61 (4H, br s), 7.0-7.6 (15H, m, Ph); m/z (FAB) 516 $\left(\mathrm{MH}^{+}\right)$, 498, 453, 408, 382; HRMS calcd. for $\mathrm{C}_{29} \mathrm{H}_{34} \mathrm{~N}_{5} \mathrm{O}_{2} \mathrm{~S}$ : 516.2433, found: 516.2436 .

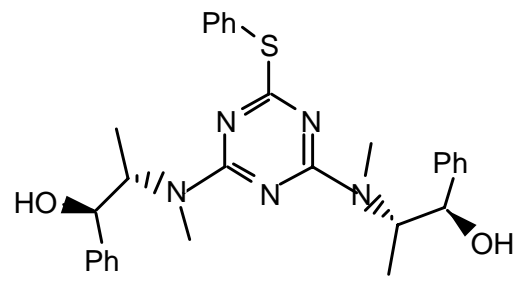

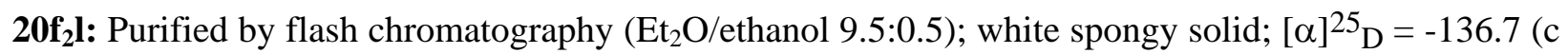
1.0, $\left.\mathrm{CHCl}_{3}\right) ; v_{\max }(\mathrm{film}) / \mathrm{cm}^{-1} 3368(\mathrm{OH}), 3109,3056,2981,2928$, 2875, 1543, 1494, 1399, 1309, 1266, 1208, 1080, 1052, 981, 803, 736, 702; $\delta_{\mathrm{H}}\left(400 \mathrm{MHz}\right.$; toluene- $\left.\mathrm{d}_{8}, 95{ }^{\circ} \mathrm{C}\right) 1.13(6 \mathrm{H}, \mathrm{d} \mathrm{J}$ 6.0, $\left.\mathrm{CHCH}_{3}\right), 2.82$ (2H, br s), 2.88 (6H, s, NMe), 3.14 (2H, q J 6.0, CHMe), 3.68 (2H, br s), 4.74 (2H, br s), 4.89 (2H, br s), $6.57(1 \mathrm{H}$, s), 6.9-7.1 (11H, m), $7.33(1 \mathrm{H}, \mathrm{s}) ; \mathrm{m} / \mathrm{z} 534\left(\mathrm{MH}^{+}\right), 516,466,426$, 358, 318; HRMS calcd. for $\mathrm{C}_{28} \mathrm{H}_{36} \mathrm{~N}_{7} \mathrm{O}_{2} \mathrm{~S}$ : 534.2651, found:

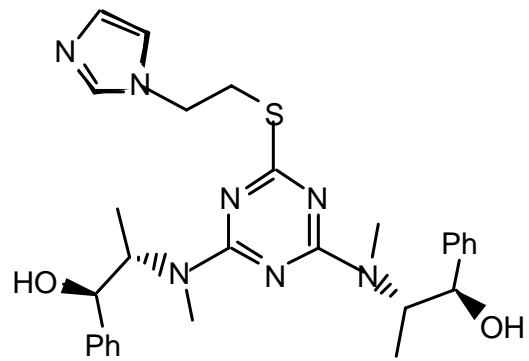
534.2649 .

20h $\mathbf{2}$ : Purified by flash chromatography (ethyl acetate/petroleum ether 40:60); white plates; m.p. 132-134 ${ }^{\circ} \mathrm{C}$ (ethanol/diethyl ether); $[\alpha]^{25} \mathrm{D}=-245.4$ (c 1.0, $\left.\mathrm{CHCl}_{3}\right) ; v_{\max }$ (nujol)/cm-1 $3200(\mathrm{OH}), 1534$, 1339, 1232, 1110, 1075, 1047, 1025, 916, 803, 762, 732, 702; $\delta_{\mathrm{H}}(400$ MHz; toluene- $\left.\mathrm{d}_{8}, 95^{\circ} \mathrm{C}\right) 1.07(4 \mathrm{H}$, br s), 1.7-1.9 (4H, m), 2.8-3.1 (4H, m), 3.65 (4H, br s), 5.12 (2H, br s), 6.47 (1H, s), 7.0-7.2 (13H, m), 7.37-7.42 (9H, m); m/z $710\left(\mathrm{MH}^{+}\right), 692,675,607,526,508,440$; HRMS calcd. for $\mathrm{C}_{42} \mathrm{H}_{44} \mathrm{~N}_{7} \mathrm{O}_{2} \mathrm{~S}$ : 710.3277, found: 710.3279 .

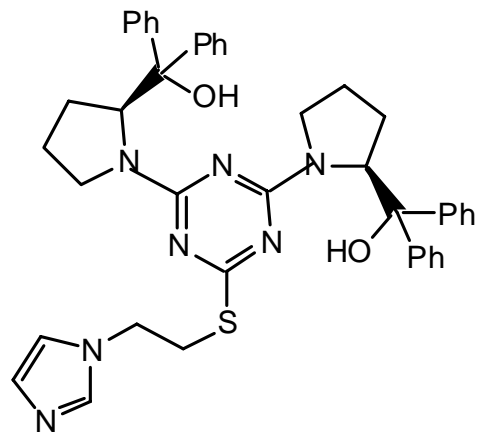

22nps: Purified by flash chromatography (hexane/ $\mathrm{CH}_{2} \mathrm{Cl}_{2}$ 7:3); colorless viscous liquid; $v_{\max }$ (film)/cm ${ }^{-1}$ 3061, 2973, 2933, 2872, 1568, 1504, 1456, 1410, 1364, 1298, 1225, 1194, 1081, 1036, 918, 807, 701, 664; $\delta_{\mathrm{H}}\left(400 \mathrm{MHz} ; \mathrm{CDCl}_{3}\right)$ 1.13, 1.17 (3H, t J 7.0, Et), 3.10, 3.13 (3H, s, NMe), 3.63, 3.65 (2H, q J 7.0, Et), 3.93 (3H, s, OMe), 4.36, 4.37 (2H, s, $\left.\mathrm{CH}_{2} \mathrm{Ph}\right), 7.21-7.31$ (3H, m, Ph), 7.40 (2H, d J 7.4, Ph); $\delta_{\mathrm{H}}(400 \mathrm{MHz}$; toluene-d8, $\left.56{ }^{\circ} \mathrm{C}\right) 0.87$ (3H, br, Et), 2.76 (3H, s, NMe), 3.55 (2H, br, Et), 3.63 (3H, s, OMe), 4.25 (2H, s, $\left.\mathrm{CH}_{2} \mathrm{Ph}\right), 7.0-7.1$ (3H, m, Ph), 7.27-7.30 (2H, m, $\mathrm{Ph})$.

25pts: Purified by flash chromatography (hexane/Et ${ }_{2} \mathrm{O}$ 9.5:0.5); $v_{\max }$ (film)/cm-1 3024, 2971, 2928, 2865, 1547, 1500, 1398, 1310, 1214, 1192, 1050, 1005; $\delta_{\mathrm{H}}\left(400 \mathrm{MHz}\right.$; toluene-d8, $\left.95{ }^{\circ} \mathrm{C}\right) 0.95\left(3 \mathrm{H}, \mathrm{t} \mathrm{J} 7.0, \mathrm{CH}_{2} \mathrm{CH}_{3}\right), 2.84(6 \mathrm{H}, \mathrm{s}$, $\mathrm{NMe}_{2}$ ), 2.86 (3H, s, NMe), 3.42 (2H, q J 7.0, $\mathrm{CH}_{2} \mathrm{CH}_{3}$ ), 4.30 (2H, s, $\left.\mathrm{CH}_{2} \mathrm{Ph}\right)$, 6.86-7.08 (3H, Ph), 7.31 (2H, d J 7.6, Ph).
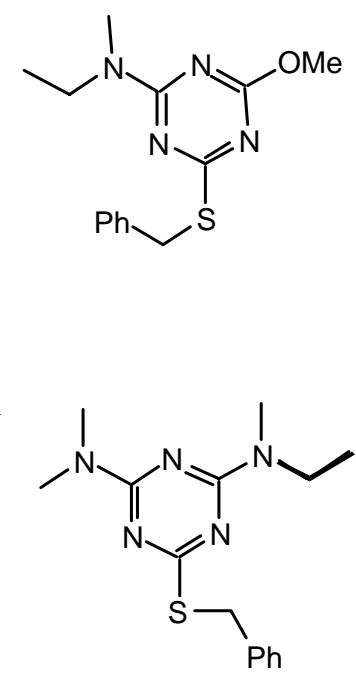
22npk: Purified by flash chromatography $\left(\mathrm{CH}_{2} \mathrm{Cl}_{2} /\right.$ hexane 6:4); colorless viscous liquid; $v_{\max }$ (film)/cm-1 3060, 2930, 2870, 1560, 1507, 1459, 1410, 1364, 1296, 1228, 1196, 1081, 1036, 916, 807, 748, 690; $\delta_{\mathrm{H}}\left(400 \mathrm{MHz} ; \mathrm{CDCl}_{3}\right)$ 0.93, 1.11 (3H, t J 7.0, Et), 2.89, 3.03 (3H, s, NMe), 3.31, 3.56 (2H, q J 7.0, Et), 3.82, 3.84 (3H, s, OMe), 7.37 (3H, br s, Ph), 7.58 (2H, br s, Ph); $\delta_{\mathrm{H}}\left(400 \mathrm{MHz}\right.$; toluene-d8, $76{ }^{\circ} \mathrm{C}$ ) 0.80 (3H, br s, Et), 2.69 (3H, br s, NMe), 3.14, 3.25 (2H, br s, Et), 3.55 (3H, s,<smiles>CCN(C)c1nc(S)nc(OC)n1</smiles>
OMe), 6.96-7.06 (3H, m, Ph), 7.49-7.50 (2H, m, Ph).

Method v): A stirred solution of triazine $\mathbf{1 9} \mathbf{g}_{2}$ (270.5 $\mathrm{mg}, 0.61 \mathrm{mmol}$ ) and 1-(3-aminopropyl)imidazole $(0.37 \mathrm{~mL}, 3.06 \mathrm{mmol})$ in dioxane $(10 \mathrm{~mL})$ under argon was refluxed during $21 \mathrm{hr}$. The solvent was evaporated in vacuo and the residue was purified by flash chromatography ( $\left.\mathrm{Et}_{2} \mathrm{O} / \mathrm{ethanol} 9: 1\right)$ to afford $\mathbf{2 0 g}_{2} \mathbf{m}$ (289.5 mg, $89 \%$ ) as a clear viscous oil, $[\alpha]^{25} \mathrm{D}=+173.4$ (c 1.1, $\left.\mathrm{CHCl}_{3}\right) ; v_{\max }($ film)/(m 13366 (NH), 3088, 3048, 2997, 2957, 2895, 1537, 1406, 1378, 1134, 1083, 1054, 918, 822, 759, 736, 702; $\delta_{\mathrm{H}}\left(400 \mathrm{MHz}\right.$; toluene-d8, $\left.95{ }^{\circ} \mathrm{C}\right) 1.01\left(6 \mathrm{H}, \mathrm{q} \mathrm{J} \mathrm{6.8,} \mathrm{CHCH}_{3}\right)$, $1.54\left(2 \mathrm{H}\right.$, quintet J 6.8, $\left.\mathrm{CH}_{2}\right), 2.94$ (6H, s, NMe), 3.05-3.08 (2H, m, $\left.\mathrm{CH}_{2} \mathrm{~N}\right), 3.39\left(2 \mathrm{H}, \mathrm{t} \mathrm{J} 6.8, \mathrm{CH}_{2} \mathrm{~N}\right), 4.54(1 \mathrm{H}, \mathrm{br}), 4.63(2 \mathrm{H}, \mathrm{d} \mathrm{J} 8.0$, $\mathrm{CHOH}), 4.86(2 \mathrm{H}$, quintet J 6.8, CHN), $6.47(1 \mathrm{H}, \mathrm{s}), 6.96-7.35(12 \mathrm{H}$, $\mathrm{m}) ; \mathrm{m} / \mathrm{z}$ (FAB) $531\left(\mathrm{MH}_{2}^{+}\right), 513,463,423,405,355,337$; HRMS calcd. for $\mathrm{C}_{29} \mathrm{H}_{39} \mathrm{~N}_{8} \mathrm{O}_{2}$ : 531.3196, found: 531.3192 .

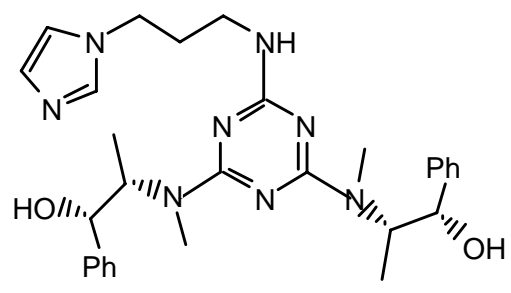

20x $\mathbf{x}_{2} \mathbf{y}$ [30,31]: Purified by flash chromatography (4:6 to 9:1 ethyl acetate/hexane), yellow liquid; $v_{\max }$ $(\mathrm{NaCl}) / \mathrm{cm}^{-1}$; 3356, 2931, 2850, 1627, 1573, 1527, 1450, 1265, 1033; $\delta_{\mathrm{H}}\left(400 \mathrm{MHz} ; \mathrm{CDCl}_{3}\right) 1.15-1.26$ (15 H, m, NH $\left.\mathrm{NH}_{2} \mathrm{CH}_{2} \mathrm{CH}_{2}\left(\mathrm{CH}_{2}\right)_{6} \mathrm{CH}_{2} \mathrm{CH}_{2} \mathrm{CO}_{2} \mathrm{CH}_{2} \mathrm{CH}_{3}\right), 1.51$ (4H, br, $\mathrm{CH}_{2} \mathrm{CO}_{2} \mathrm{Et} / \mathrm{NH}_{2} \mathrm{CH}_{2}$ ), 2.27 (2H, t, $\mathrm{J}=8, \mathrm{CH}_{2} \mathrm{CO}_{2} \mathrm{Et}$ ), 3.04 (2H, m, $\mathrm{NH}_{2} \mathrm{CH}_{2}$ ), 3.35 (6H, bs, $\mathrm{OCH}_{3}$ ), 3.52 (8H, m, $\mathrm{CH}_{2} \mathrm{CH}_{2} \mathrm{OMe}$ ), $4.11\left(2 \mathrm{H}, \mathrm{q}, \mathrm{J}=8,-\mathrm{CO}_{2} \mathrm{CH}_{2} \mathrm{CH}_{3}\right.$ ); $\delta_{\mathrm{C}}\left(\mathrm{CDCl}_{3}\right)$ 14.21, 24.93, 26.83, 29.08, 29.18, 29.26, 29.33, 29.43, 29.57, 34.34, 40.42, 40.73, 58.75, 60.10, 71.24, 164.42, 173.87; m/z (CI) $455\left(\mathrm{MH}^{+}\right), 453,423$; HRMS calcd. for $\mathrm{C}_{22} \mathrm{H}_{43} \mathrm{~N}_{6} \mathrm{O}_{4}$ : 455.334, found: 455.333 .

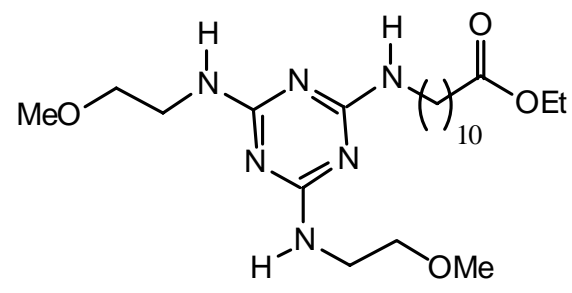

Method vi): To a stirred solution of anhydrous methanol (0.23 mL, $5.77 \mathrm{mmol}$ ) in anhydrous THF (30 $\mathrm{mL}$ ) at room temperature under argon was added sodium hydride (277 mg, 50 \% oil dispersion, 5.77 $\mathrm{mmol}$ ). After $2.5 \mathrm{hr}$ the mixture was refluxed for $15 \mathrm{~min}$., cooled to $0{ }^{\circ} \mathrm{C}$, added at once cyanuric chloride (532 mg, $2.89 \mathrm{mmol}$ ) and the reaction mixture was allowed to warm to room temperature and stirred for $2 \mathrm{hr}$. Diisopropylethylamine $(0.60 \mathrm{~mL}, 3.46 \mathrm{mmol})$ and 2-(methylamino)ethanol $(0.26 \mathrm{~mL}$, $3.17 \mathrm{mmol}$ ) were added and the mixture was stirred for 2.5 days. The mixture was partitioned between $\mathrm{Et}_{2} \mathrm{O}(70 \mathrm{~mL})$ and saturated aqueous sodium chloride $(70 \mathrm{~mL})$. The aqueous phase was extracted with $\mathrm{Et}_{2} \mathrm{O}(2 \times 70 \mathrm{~mL})$, the combined organic layers were dried $\left(\mathrm{MgSO}_{4}\right)$, filtered, evaporated to dryness and purified by flash chromatography ( $\mathrm{Et}_{2} \mathrm{O} /$ ethyl acetate $1: 1$ to $0: 1$ ) to afford in order of elution 21ni (173.3 mg, $28 \%$ ) as white solid; and $\mathbf{2 4} \mathbf{n}_{\mathbf{2}} \mathbf{i}$ (245.7 $\mathrm{mg}, 40 \%$ ) as a white solid; 
21ni: White needles; m.p. 141-142 ${ }^{\circ} \mathrm{C}$ (ethyl acetate/hexane); $v_{\max }$ (film)/(cm ${ }^{-1} 3364$ (OH), 3234 (OH), 3008, 2960, 2875, 1606, 1508, 1416, 1373, 1292, 1205, 1068, 1028, 799; $\delta_{\mathrm{H}}\left(400 \mathrm{MHz}\right.$; toluene-d8, $\left.95^{\circ} \mathrm{C}\right) 2.80$ (3H, s, NMe), 3.27 (2H, br s), 3.36 (2H, m), 3.57 (3H, s, OMe); m/z (FAB) $219\left(\mathrm{MH}^{+}\right), 201,187,154,136$, 107; HRMS calcd. for $\mathrm{C}_{7} \mathrm{H}_{12} \mathrm{~N}_{4} \mathrm{O}_{2} \mathrm{Cl}$ : 219.0649, found: 219.0660; Anal. calcd. for $\mathrm{C}_{7} \mathrm{H}_{11} \mathrm{~N}_{4} \mathrm{O}_{2} \mathrm{Cl}$ : $\mathrm{H} 5.07 \mathrm{C} 38.45$ N 25.62 \%. Found: $\mathrm{H} 4.82 \mathrm{C}$

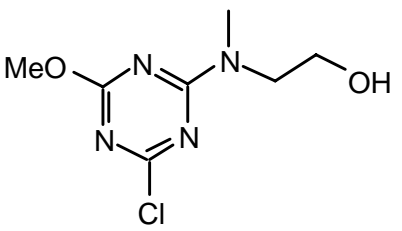
38.45 N $25.45 \%$.

24n $\mathbf{i}$ : White needles; m.p. 80.5-81 ${ }^{\circ} \mathrm{C}$ (ethyl acetate/hexane); $v_{\max }$ (film)/cm-1 3185 (OH), 3003, 2955, 2870, 1612, 1544, 1469, 1360, 1310, 1239, 1136, 1058, 808; $\delta_{\mathrm{H}}(400$ $\left.\mathrm{MHz} ; \mathrm{CDCl}_{3}\right) 2.84$ (3H, s, NMe), 3.33 (2H, t J 5.2), 3.54 (2H, br s), 3.62 (3H, OMe), 3.65 (3H, s, OMe); m/z (FAB) $215\left(\mathrm{MH}^{+}\right)$, 183, 169, 154, 136; HRMS calcd. for $\mathrm{C}_{8} \mathrm{H}_{15} \mathrm{~N}_{4} \mathrm{O}_{3}$ : 215.1144, found: 215.1160; Anal. calcd. for $\mathrm{C}_{8} \mathrm{H}_{14} \mathrm{~N}_{4} \mathrm{O}_{3}$ : H 6.59 C 44.85 N 26.15 \%. Found: H 6.88 C 44.90 N $26.88 \%$.

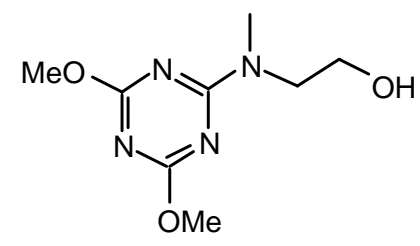

Method vii): To a stirred solution of cyanuric chloride (204.6 mg, $1.11 \mathrm{mmol}$ ) and diisopropylethylamine $(0.43 \mathrm{ml}, 2.44 \mathrm{mmol})$ in anhydrous $\mathrm{CH}_{2} \mathrm{Cl}_{2}(11 \mathrm{~mL})$ at $0{ }^{\circ} \mathrm{C}$ under argon, was added dropwise (3 min.) 2-(methylamino)ethanol (89 $\mu \mathrm{L}, 1.11 \mathrm{mmol}$ ) [32]. After $15 \mathrm{~min}$. the reaction mixture was allowed to warm to room temperature, stirred for $45 \mathrm{~min}$ and was added dropwise via cannula a solution of (2-methoxy-ethyl)methylamine (j) [33] (1.11 mmol) in ethanol (6 mL) [34]. After $18 \mathrm{hr}$ the solvent was evaporated to dryness and purified by flash chromatography (hexane/Et ${ }_{2} \mathrm{O}$ 1:1); to afford 23ij (244 mg, $80 \%$ ) as a viscous colorless liquid; $v_{\max }$ (film)/cm-1 3417 (OH), 2940, 2883, 1577, 1492, 1401, 1310, 1202, 1117, 1049, 998, 969, 799; $\delta_{\mathrm{H}}\left(400 \mathrm{MHz}\right.$; toluene-d8, $\left.95{ }^{\circ} \mathrm{C}\right) 2.85$ (3H, s), 2.93 (3H, s), 3.06 (3H, s), 3.30-3.34 (4H, m), 3.47 (4H, t J 4.7); m/z (FAB) 276 $\left(\mathrm{MH}^{+}\right)$, 258, 244, 230, 217, 200 149; HRMS calcd. for $\mathrm{C}_{10} \mathrm{H}_{19} \mathrm{~N}_{5} \mathrm{O}_{2} \mathrm{Cl}: 276.1227$, found: 276.1220.

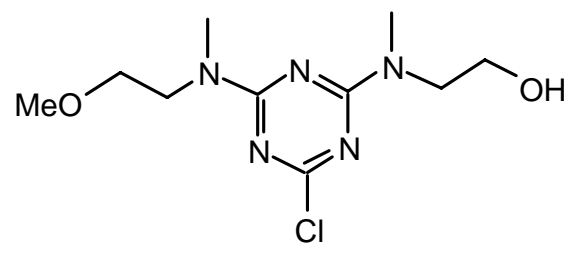

23it: Purified by flash chromatography ( $\mathrm{Et}_{2} \mathrm{O} /$ hexane 6:4); white plates; m.p. 101.5-102 ${ }^{\circ} \mathrm{C}$ (diethyl ether / hexane); $v_{\max }$ (film)/cm-1 $3400(\mathrm{OH}), 2938,1574,1526,1489,1402,1310,1203,1045,993$, 861, 841, 798; $\delta_{\mathrm{H}}\left(400 \mathrm{MHz}\right.$; toluene-d8, 84 $\left.{ }^{\circ} \mathrm{C}\right) 2.93\left(6 \mathrm{H}, \mathrm{s}, \mathrm{NMe}_{2}\right), 3.05$ (3H, s, NMe), 3.54 (2H, br s, $\left.\mathrm{CH}_{2} \mathrm{~N}\right), 3.68\left(2 \mathrm{H}\right.$, br s, $\left.\mathrm{CH}_{2} \mathrm{OH}\right) ; \mathrm{m} / \mathrm{z}(\mathrm{FAB})$ $232\left(\mathrm{MH}^{+}\right), 214,200,186,154,136$; HRMS calcd. for $\mathrm{C}_{8} \mathrm{H}_{15} \mathrm{~N}_{5} \mathrm{OCl}$ : 232.0965, found: 232.0960; Anal. calcd. for $\mathrm{C}_{8} \mathrm{H}_{14} \mathrm{~N}_{5} \mathrm{OCl}$ : H 6.09 C 41.47 N 30.23 \%. Found: H 6.02 C 41.46 N 30.32 \%.

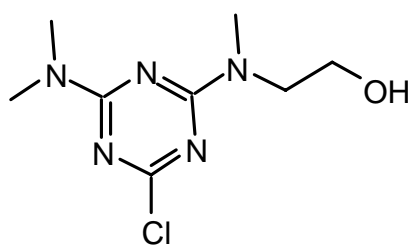

23jt: Purified by flash chromatography ( $\mathrm{Et}_{2} \mathrm{O} /$ hexane 6:4); white plates; m.p. $51-53{ }^{\circ} \mathrm{C}$ (hexane); $v_{\max }$ (film)/cm-1 2940, 2883, 2832, 1571, 1520, 1492, 1395, 1304, 1196, 1111, 1054, 992, 969, 839, 793; $\delta_{\mathrm{H}}\left(400 \mathrm{MHz}\right.$; toluene-d $\mathrm{d}_{8}$ ) 2.59, 2.62 (3H, s, NMe), 2.72 (3H, s, NMe), 2.91, 2.93 (3H, s, NMe), 2.98, 3.02 (3H, s, OMe), 3.24 (2H, t J 5.2, $\mathrm{CH}_{2} \mathrm{~N}$ ), 3.41, 3.49 (2H, t J 5.2, $\left.\mathrm{CH}_{2} \mathrm{OMe}\right) ; \mathrm{m} / \mathrm{z}$ (FAB) $246\left(\mathrm{MH}^{+}\right), 230,214,200,187$; HRMS calcd. for $\mathrm{C}_{9} \mathrm{H}_{17} \mathrm{~N}_{5} \mathrm{OCl}$ :<smiles>COCCN(C)c1nc(Cl)nc(N(C)C)n1</smiles> 
246.1122, found: 246.1110 .

21np: Purified by flash chromatography (ethyl acetate/hexane 9:1); white needles; m.p. 62-62.5 $\mathrm{C}$ (hexane); $v_{\max }$ (film)/cm-1 2974, 2930, 1571, 1497, 1372, 1293, 1032; $\delta_{\mathrm{H}}(400$ MHz; $\left.\mathrm{CDCl}_{3}\right) 1.19$ (3H, 3 J 7.1, $\left.\mathrm{NCH}_{2} \mathrm{CH}_{3}\right), 3.14,3.16$ (3H, s, NMe), 3.66 (2H, quint J 7.2, $\left.\mathrm{NCH}_{2} \mathrm{CH}_{3}\right), 3.96$ (3H, s, OMe); m/z (FAB) $203\left(\mathrm{MH}^{+}\right), 187,173$, 154, 136, 110; HRMS calcd. for $\mathrm{C}_{7} \mathrm{H}_{12} \mathrm{~N}_{4} \mathrm{OCl}$ : 203.0700, found: 203.0720; Anal. calcd. for $\mathrm{C}_{7} \mathrm{H}_{11} \mathrm{~N}_{4} \mathrm{OCl}$ : H $5.47 \mathrm{C} 41.49 \mathrm{~N} 27.65$ \%. Found: $\mathrm{H} 5.64 \mathrm{C}$

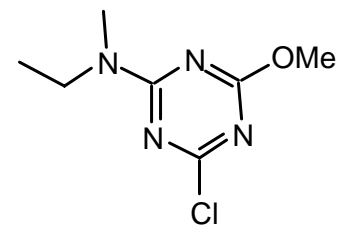
41.81 N $28.03 \%$.

23pt: Purified by flash chromatography $\left(\mathrm{Et}_{2} \mathrm{O} /\right.$ hexane 9.4:0.6); white solid $\left(7^{\circ} \mathrm{C}\right) ; v_{\max }$ (film)/cm-1 2974, 2934, 2877, 1577, 1497, 1406, 1316, 1202, 1054, 1003, 969, 941, 844, 804; $\delta_{\mathrm{H}}\left(400 \mathrm{MHz} ; \mathrm{CDCl}_{3}\right) 1.11(3 \mathrm{H}, \mathrm{t} \mathrm{J} 7.0$, $\left.\mathrm{CH}_{2} \mathrm{CH}_{3}\right), 3.04,3.06,3.09(9 \mathrm{H}, \mathrm{s}), 3.57\left(2 \mathrm{H}\right.$, quint J 7.0, $\left.\mathrm{CH}_{2} \mathrm{CH}_{3}\right), \delta_{\mathrm{H}}(400$ $\mathrm{MHz}$; toluene- $\left.\mathrm{d}_{8}, 95{ }^{\circ} \mathrm{C}\right) 0.91$ (3H, t J 6.4, $\left.\mathrm{CH}_{2} \mathrm{CH}_{3}\right), 2.77$ (6H, s, $\left.\mathrm{NMe}_{2}\right), 2.97$

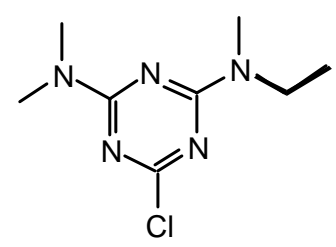
(3H, s, NMe), 3.34 (2H, br s, $\left.\mathrm{CH}_{2} \mathrm{CH}_{3}\right)$;

23ps: Purified by flash chromatography (hexane/ $\mathrm{CH}_{2} \mathrm{Cl}_{2} 1: 1$ ); white plates; m.p. $47.5-48{ }^{\circ} \mathrm{C}$ (hexane); $v_{\max }(\mathrm{film}) / \mathrm{cm}^{-1}$ 3031, 2974, 2929, 2877, 1577, 1475, 1412, 1344, 1327, 1236, 1168, 1032, 912, 850, 793, 736; $\delta_{\mathrm{H}}\left(400 \mathrm{MHz} ; \mathrm{CDCl}_{3}\right) 1.14,1.18$ (3H, t J 7.0, Et), 3.128, 3.135 (3H, s, NMe); 0.64 (2H, q J 7.0, Et), 4.33, 4.35 (2H, s, $\left.\mathrm{CH}_{2} \mathrm{Ph}\right), 7.25-7.33$ (3H, m, Ph), 7.39 (2H, d J 7.0 Ph); m/z (FAB) $295\left(\mathrm{MH}^{+}\right), 279,261,243$, 217, 205, 169; HRMS calcd. for $\mathrm{C}_{13} \mathrm{H}_{16} \mathrm{~N}_{4} \mathrm{SCl}$ : 295.0784, found: 295.0770; Anal. calcd. for $\mathrm{C}_{13} \mathrm{H}_{15} \mathrm{~N}_{4} \mathrm{SCl}$ : H $5.13 \mathrm{C} 52.97$ N 19 \%. Found: H $5.18 \mathrm{C}$

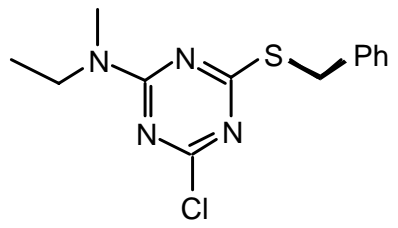
52.98 N 19.00\%.

23pu: Purified by flash chromatography (hexane/ $\mathrm{CH}_{2} \mathrm{Cl}_{2} 1: 1$ to 0:1); prisms; m.p. $44-45{ }^{\circ} \mathrm{C}$ (hexane); $v_{\max }(\mathrm{film}) / \mathrm{cm}^{-1}$ 2974, 2877, 1566, 1412, 1350, 1321, 1300, 1191, 1168, 1009, 969, 827, 804; $\delta_{\mathrm{H}}$ (400 MHz; $\left.\mathrm{CDCl}_{3}\right) 1.14$ (3H, t J 7.0, Et), 1.93 (4H, br s, $\left.\left(\mathrm{CH}_{2}\right)_{2}\right)$, 3.08, 3.09 (3H, s, NMe), 3.52-3.61 (4H, m, NCH $), 3.56(2 \mathrm{H}, \mathrm{q} \mathrm{J} 7.0, \mathrm{Et}) ; \delta_{\mathrm{H}}(400$ MHz; toluene-d $\left.8,50{ }^{\circ} \mathrm{C}\right) 0.94(3 \mathrm{H}, \mathrm{br} \mathrm{s}, \mathrm{Et}), 1.43\left(4 \mathrm{H}, \mathrm{br} \mathrm{s},\left(\mathrm{CH}_{2}\right)_{2}\right), 2.82$ (3H, br s, NMe), 3.23 (2H, br s), 3.33-3.39 (4H, m); m/z (FAB) $242\left(\mathrm{MH}^{+}\right)$, 226, 212, 200; HRMS calcd. for $\mathrm{C}_{10} \mathrm{H}_{17} \mathrm{~N}_{5} \mathrm{Cl}$ : 242.1172, found: 242.1160 . Anal. calcd. for $\mathrm{C}_{10} \mathrm{H}_{16} \mathrm{~N}_{5} \mathrm{Cl}$ : H $6.67 \mathrm{C} 49.69 \mathrm{~N} 28.97$ \%. Found: H $6.94 \mathrm{C}$<smiles>CCN(C)c1nc(Cl)nc(N2CCCC2)n1</smiles>
49.71 N $29.50 \%$.

23qv: Purified by flash chromatography (hexane/ $\mathrm{CH}_{2} \mathrm{Cl}_{2}$ 6:4); white plates; m.p. 145.5-146 ${ }^{\circ} \mathrm{C}$ (cyclohexane); $v_{\max }$ (film)/cm-1 3417 (NH), 3264 (NH), 3065, 3025, 2974, 2929, 1577, 1509, 1395, 1287, 1168, 975, 907, 804, 731, 702; $\delta_{\mathrm{H}}\left(400 \mathrm{MHz}\right.$; toluene-d $\left.8,95{ }^{\circ} \mathrm{C}\right) 1.11(9 \mathrm{H}, \mathrm{s}, t-\mathrm{Bu})$, 2.54 (3H, s, NMe), 6.27 (1H, s, NH), 6.95-7.21 (15H, m, Ph); m/z (FAB)<smiles>CN(c1nc(Cl)nc(NC(c2ccccc2)c2ccccc2)n1)C(C)(C)C</smiles>
$458\left(\mathrm{MH}^{+}\right)$, 401, 380, 324, 243; HRMS calcd. for $\mathrm{C}_{27} \mathrm{H}_{29} \mathrm{~N}_{5} \mathrm{Cl}$ : 458.2111, 
found: 458.2130; Anal. calcd. for $\mathrm{C}_{27} \mathrm{H}_{28} \mathrm{~N}_{5} \mathrm{Cl}$ : H 6.16 C 70.81 N 15.29 \%. Found: H 6.21 C $70.81 \mathrm{~N}$ $15.29 \%$.

23xy [28]: Recrystallized from ethyl acetate/hexane, yellow solid m.p 114-116 ${ }^{\circ} \mathrm{C} ; v_{\max }(\mathrm{NaCl}) / \mathrm{cm}^{-1}$; 3247, 3109, 2931, 2854, 1735, 1643, 15558, 1411, 1119; $\delta_{\mathrm{H}}\left(400 \mathrm{MHz} ; \mathrm{CDCl}_{3}\right)$ 1.18-1.22 (15 H, m, $\left.\mathrm{NH}_{2} \mathrm{CH}_{2} \mathrm{CH}_{2}\left(\mathrm{CH}_{2}\right)_{6} \mathrm{CH}_{2} \mathrm{CH}_{2} \mathrm{CO}_{2} \mathrm{CH}_{2} \mathrm{CH}_{3}\right), 1.55$ (4H, br, $\left.\mathrm{CH}_{2} \mathrm{CO}_{2} \mathrm{Et} / \mathrm{NH}_{2} \mathrm{CH}_{2}\right), 2.23$ (2H, t, J=8 $\mathrm{CH}_{2} \mathrm{CO}_{2} \mathrm{Et}$ ), 3.36-3.30 (5H, m NH $\left.\mathrm{CH}_{2} / \mathrm{OCH}_{3}\right), 3.49$ (2H, t, J=4 $\mathrm{CH}_{2} \mathrm{CH}_{2} \mathrm{OMe}$ ), 3.57 (2H, t, J=4 $\mathrm{CH}_{2} \mathrm{CH}_{2} \mathrm{OMe}$ ), 4.07 (2H, q, J= 8 $\left.-\mathrm{CO}_{2} \mathrm{CH}_{2} \mathrm{CH}_{3}\right) ; \delta_{\mathrm{C}}\left(\mathrm{CDCl}_{3}\right)$ 14.12, 24.82, 26.71, 28.97, 29.09, 29.22, 29.33, 34.22, 40.43, 40.79, 58.68, 60.00, 70.67, 165,44, 167.74, 173.72; m/z (CI) $416\left(\mathrm{MH}^{+}\right)$, 380; HRMS calcd. for $\mathrm{C}_{19} \mathrm{H}_{35} \mathrm{ClN}_{5} \mathrm{O}_{3}$ : 416.242, found: 416.244 .

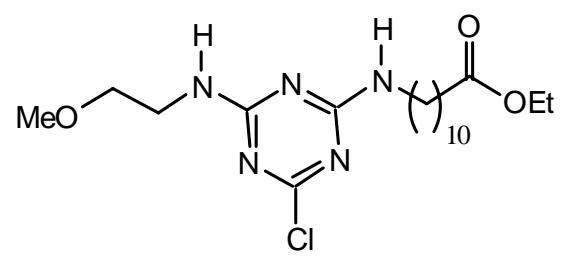

Method viii): To a stirred solution of $t$-butyl-alcohol $(294 \mu \mathrm{L}, 3.08 \mathrm{mmol})$ in anhydrous hexane (2 mL) under argon at room temperature was added dropwise $n$-butyllithium (2.37 mL, $3.08 \mathrm{mmol}, 1.3 \mathrm{M}$ ). The above mixture was added dropwise via cannula to a stirred solution of cyanuric chloride (567.5 $\mathrm{mg}, 3.08 \mathrm{mmol})$ in anhydrous THF $(20 \mathrm{~mL})$ under argon at room temperature. After $20 \mathrm{~min}$ diisopropylethylamine $(590 \mu \mathrm{L}, 3.39 \mathrm{mmol}$ ) and $N$-ethylmethylamine (264 $\mu \mathrm{L}, 3.08 \mathrm{mmol}$ ) were added. After 2.5 days the mixture was partitioned between $\mathrm{CH}_{2} \mathrm{Cl}_{2}(40 \mathrm{~mL})$ and saturated aqueous ammonium chloride (30 mL). The aqueous phase was extracted with $\mathrm{CH}_{2} \mathrm{Cl}_{2}(2 \times 30 \mathrm{~mL})$, the combined organic layers were dried $\left(\mathrm{MgSO}_{4}\right)$, filtered, evaporated to dryness and purified by flash chromatography (hexane/ $\mathrm{CH}_{2} \mathrm{Cl}_{2}$ 7:3 to $1: 1$ ) to afford 210 p (413.2 mg, 55 \%) as a viscous colorless liquid; $v_{\max }(\mathrm{film}) / \mathrm{cm}^{-1}$ 2980, 2940, 1571, 1293, 1168, 1077, 998, 941, 804; $\delta_{\mathrm{H}}\left(400 \mathrm{MHz} ; \mathrm{CDCl}_{3}\right) 1.18(3 \mathrm{H}, \mathrm{t} \mathrm{J} 7.0, \mathrm{Et}), 1.59,1.60$ (9H, s, $\mathrm{OCMe}_{3}$ ), 3.11, 3.14 (3H, s, NMe), 3.62, 3.65 (2H, q J 7.0, Et); $\delta_{\mathrm{H}}(400$ MHz; toluene-d $\left.8,67{ }^{\circ} \mathrm{C}\right) 0.83$ (3H, t J 7.2, Et), 1.45 (9H, s, OCMe3), 2.70 (3H, s, NMe), 3.25 (2H, br , Et); m/z (FAB) $245\left(\mathrm{MH}^{+}\right), 227,212,189$; HRMS calcd. for $\mathrm{C}_{10} \mathrm{H}_{18} \mathrm{~N}_{4} \mathrm{OCl}$ : 245.1169, found: 245.1150 .

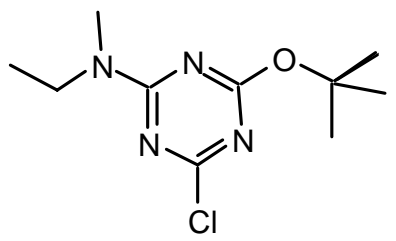

Method ix: To a stirred solution of cyanuric chloride (158.1 mg, $0.86 \mathrm{mmol}$ ), DMAP (catalytic amount) and diisopropylethylamine $(493 \mu \mathrm{L}, 2.83 \mathrm{mmol})$ in anhydrous $\mathrm{CH}_{2} \mathrm{Cl}_{2}(8 \mathrm{~mL})$ at room temperature under argon, was added dropwise anhydrous methanol ( $35 \mu \mathrm{L}, 0.86 \mathrm{mmol}$ ). After $2.5 \mathrm{hr}$ 5,6,11,12-tetrahydro-dibenzo[b,f]azoic hydro-chloride (232 mg, $0.94 \mathrm{mmol}$ ) was added at once and the mixture was refluxed during $14 \mathrm{hr}$. The solvent was evaporated to dryness and purified by flash chromatography (hexane/ $\mathrm{CH}_{2} \mathrm{Cl}_{2} 4: 6$ to 3:7) to afford $21 \mathrm{nr}$ (273.6 mg, 91 \%) as white cubes; m.p. 163-164 oC (cyclohexane); $v_{\max }\left(\right.$ film) $/ \mathrm{cm}^{-1}$ 3065, 3025, 2963, 2940, 1566, 1509, 1378, 1293, 1225, 1066, 1043, 975, 816, 770, 736; $\delta_{\mathrm{H}}(400 \mathrm{MHz}$; toluene- $\left.\mathrm{d}_{8}\right)$ 2.82-2.89 (2H, m), 3.04-3.14 (2H, m), 3.68, 4.05 (3H, s, OMe), 4.39 (1H, d J 15.0), 5.86 (1H, t J 15.0), 7.04-7.19 (8H, m, Ar); m/z (FAB) 353 $\left(\mathrm{MH}^{+}\right)$, 337, 317, 259, 231, 206; HRMS calcd. for $\mathrm{C}_{19} \mathrm{H}_{18} \mathrm{~N}_{4} \mathrm{OCl}$ : 353.1169, found: 353.1150; Anal. calcd. for $\mathrm{C}_{19} \mathrm{H}_{17} \mathrm{~N}_{4} \mathrm{OCl}$ : H 4.86 C $64.68 \mathrm{~N} 15.88$ \%. Found: H 4.83 C 64.67 N $15.84 \%$.

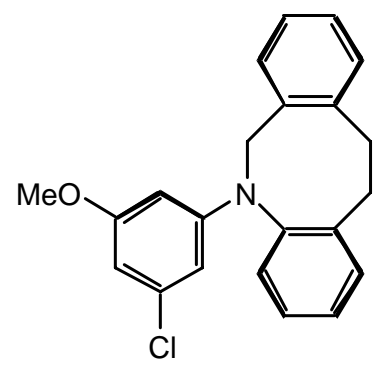


21nq: Purified by flash chromatography $\left(\mathrm{CH}_{2} \mathrm{Cl}_{2}\right.$ /hexane 7:3); colorless needles; m.p. 156-157 ${ }^{\circ} \mathrm{C}$ (hexane); $v_{\max }(\mathrm{film}) / \mathrm{cm}^{-1} 3406(\mathrm{NH}), 3258(\mathrm{NH}), 3093,3059,3031,1566$, $1475,1395,1372,1293,1270,1202,1111,1060,1037,816,742,702 ; \delta_{\mathrm{H}}(400$ MHz; $\left.\mathrm{CDCl}_{3}\right) 3.23$ (3H, s, OMe), 6.91 (1H, s, NH), 7.24 (15H, br s, Ph); m/z (FAB) $403\left(\mathrm{MH}^{+}\right)$, 325, 243; HRMS calcd. for $\mathrm{C}_{23} \mathrm{H}_{20} \mathrm{~N}_{4} \mathrm{OCl}$ : 403.1326, found: 403.1310; Anal. calcd. for $\mathrm{C}_{23} \mathrm{H}_{19} \mathrm{~N}_{4} \mathrm{OCl}$ : $\mathrm{H} 4.75 \mathrm{C} 68.57 \mathrm{~N} 13.91 \%$.

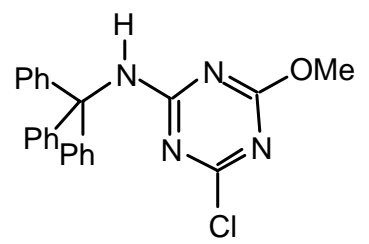
Found: H 4.75 C 68.59 N $13.97 \%$.

Method x: To a stirred solution of $t$-butyl alcohol $(77.5 \mu \mathrm{L}, 0.81 \mathrm{mmol})$ in anhydrous hexane (2 $\mathrm{mL})$ under argon at room temperature was added dropwise $n$-butyllithium $(570 \mu \mathrm{L}, 0.81 \mathrm{mmol}, 1.4 \mathrm{M})$. The above mixture was added dropwise via cannula to a stirred solution of cyanuric chloride (149.5 mg, $0.81 \mathrm{mmol})$ in anhydrous THF (8 mL) under argon at room temperature. After 20 min was added DMAP (catalytic amount), diisopropylethylamine $(169 \mu \mathrm{L}, 0.97 \mathrm{mmol})$ and triethylamine (231 mg, $0.81 \mathrm{mmol}$ ) and the mixture was refluxed during 5 days. The mixture was partitioned between $\mathrm{Et}_{2} \mathrm{O}$ $(25 \mathrm{~mL})$ and saturated aqueous ammonium chloride $(25 \mathrm{~mL})$. The aqueous phase was extracted with diethyl ether ( $2 \times 25 \mathrm{~mL}$ ), the combined organic layers were dried $\left(\mathrm{MgSO}_{4}\right)$, filtered, evaporated to dryness, purified by flash chromatography (hexane/dichloromethane 1:1) and preparative TLC (hexane/ethyl acetate 9:1) to afford $210 q\left(35.3 \mathrm{mg}, 10 \%\right.$ ) as a slightly yellow spongy solid, $v_{\max }$ (film)/cm 3406 (NH), 3247 (NH), 3088, 3065, 3031, 2963, 2934, 2872, 1554, 1441, 1293, 1168, 1060, 1036, 986, 912, 815, 736, 702; $\delta_{\mathrm{H}}\left(400 \mathrm{MHz} ; \mathrm{CDCl}_{3}\right) 0.94(9 \mathrm{H}, \mathrm{s}, t-$ $\mathrm{Bu}), 6.87(1 \mathrm{H}, \mathrm{s}, \mathrm{NH}), 7.07-7.26(15 \mathrm{H}, \mathrm{m}, \mathrm{Ph}) ; \mathrm{m} / \mathrm{z}(\mathrm{FAB}) 445\left(\mathrm{MH}^{+}\right)$, 388, 367, 311, 289, 275, 259; HRMS calcd. for $\mathrm{C}_{26} \mathrm{H}_{26} \mathrm{~N}_{4} \mathrm{OCl}$ : 445.1795, found: 445.1780; Anal. calcd. for $\mathrm{C}_{26} \mathrm{H}_{25} \mathrm{~N}_{4} \mathrm{OCl}$ : $\mathrm{H} 5.66 \mathrm{C} 70.18 \mathrm{~N} 12.59$ \%. Found: H 5.68 C 70.09 N $12.30 \%$.

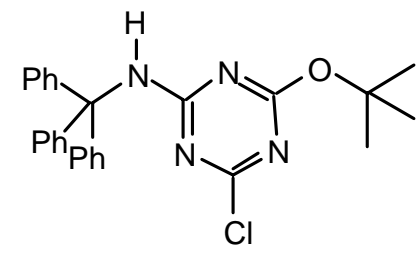

\section{References and Notes}

1. (a) Hollink, E.; Simanek, E. E.; Bergbreiter, D. E. Tetrahedron Lett. 2005, 46, 2005-2008, and references cited therein; (b) Herrera, A.; Martínez-Alvarez, R. P.; Chioua, M.; Chioua, R. Synthesis 2004, 503-505, and refrences cited therein; (c) Barton, B.; Gouwns, S.; Schaefer, M. C.; Zeelie, B. Org. Process Res. Dev. 2003, 7, 1071-1076 and references cited therein; (d) Azenha, M. E. D. G.; Burrows, H. D.; Canle, M. L.; Coimbra, R.; Fernández, M. I.; García, M. V.; Rodrigues, A. E.; Santaballa, J. A.; Steenken, S. Chem. Comm. 2003, 112-113; (e) Draber, W.; Tietjen, K.; Kluth, J. F.; Trebst, A. Angew. Chem. Int. Ed. 1991, 30, 1621-1633; (f) Quirke, J. M. E. In Comprehensive Heterocyclic Chemistry, Katritzky, A. R.; Rees, C. W., eds.; Pergamon Press: London, 1984; 3, 457-530.

2. (a) Hedayatullah, M.; Lion, C.; Slimane, A. B.; Da Conceição, L.; Nachawati, I. Heterocycles 1999, 51, 1891-1896 and references cited therein. For some other examples of 1,3,5-triazines which present important biological properties see: Antitumor activity: (b) Garaj, V.; Puccetti, L.; Fasolis, G.; Winum, J.-Y.; Montero, J.-L.; Scozzafava, A.; Vullo, D.; Innocenti, A.; Supuran, C. T. Bioorg. Med. Chem. Lett. 2005, 15, 3102-3108; (c) Saczewski, F.; Brzozowski, Z. Eur. J. Med. 
Chem. 2002, 37, 709-720; (d) Saczewski, F.; Brzozowski, Z.; Gdaniec, M. Eur. J. Med. Chem. 2000, 35, 1053-1064; Estrogen receptor: (e) Henke, B. R.; Consler, T. G.; Go, N.; Hale, R. L.; Hohman, D. R.; Jones, S. A.; Lu, A. T.; Moore, L. B.; Moore, J. T.; Orband-Miller, L. A.; Robinett, R. G.; Shearin, J.; Spearing, P. K.; Stewart, E. L.; Turnbull, P. S.; Weaver, S. L.; Williams, S. P.; Wisely, G. B.; Lambert, M. H. J. Med. Chem. 2002, 45, 5492-5505; Cyclindependent kinase inhibitors: (f) Kuo, G.-H.; DeAngelis, A.; Emanuel, S.; Wang, A.; Zhang, Y.; Connolly, P. J.; Chen, X.; Gruninger, R. H.; Rugg, C.; Fuentes-Pesquera, A.; Middleton, S. A.; Jolliffe, L.; Murray, W. V. J. Med. Chem. 2005, 48, 5435-4546; Multidrug resistance reversal agent: (g) Robert, J.; Jarry, C. J. Med. Chem. 2003, 46, 4805-4817 (perspective article).

3. (a) Matsuno, T.; Kato, M.; Sasahara, H.; Watanabe, T.; Inaba, M.; Takahashi, M.; Yaguchi, S.-I., Yoshioka, K.; Sakato, M.; Kawashima, S. Chem. Pharm. Bull. 2000, 48, 1778-1781; (b) Matsuno, T.; Kato, M.; Tsuchida, Y.; Takahashi, M.; Yaguchi, S.; Terada, S. Chem. Pharm. Bull. 1997, 45, 291-296 and references cited therein.

4. Ramurthy, S.; Miller, M. J. J. Org. Chem. 1996, 61, 4120-4124.

5. Whitten, J. P.; Xie, Y. F.; Erickson, P. E.; Webb, T. R.; De Souza, E. B.; Grigoriadis, D. E.; McCarthy, J. R. J. Med. Chem. 1996, 39, 4354-4357.

6. Hasegawa, Y.; Yanagisawa, T.; Okui, Y.; Sato, T.; Hosaka, K.; Chin, M.; Mitsuhashi, H. Chem. Pharm. Bull. 1991, 39, 3180-3182.

7. He, L.; Gilligan, P. J.; Zaczek, R.; Fitzgerald, L. W.; McElroy, J.; Shen, H-S. L.; Saye, J. A.; Kalin, N. H.; Shelton, S.; Christ, D.; Trainor, G.; Hartig, P. J. Med. Chem. 2000, 43, 449-456.

8. Klenke, B.; Stewart, M.; Barrett, M. P.; Brun, R.; Gilbert, I. H. J. Med. Chem. 2001, 44, 34403452.

9. (a) Khersonsky, S. M., Jung, D.-W.; Kang, T.-W.; Walsh, D. P.; Moon, H.-S., Jo, H.; Jacobson, E. M.; Shetty, V.; Neubert, T. A.; Chang, Y.-T. J. Am. Chem. Soc. 2003, 125, 11804-11805; (b) Scharn, D.; Germeroth, L.; Schneider-Mergener, J.; Wenschuh, H. J. Org. Chem. 2001, 66, 507513; (c) Scharn, D.; Wenschuh, H.; Reineke, U.; Schneider-Mergener, J.; Germeroth, L. J. Comb. Chem. 2000, 2, 361-369 ; (d) Masquelin, T.; Meunier, N.; Gerber, F.; Rossé, G. Heterocyles 1998, 48, 2489-2505 ; (e) Falorni, M.; Giacomelli, G.; Mameli, L.; Porcheddu, A. Tetrahedron Lett. 1998, 39, 7607-7610; (f) Stanková, M.; Lebl, M. Mol. Divers. 1996, 2, 75-80; (g) Gustafson, G. R.; Baldino, C. M.; O’Donnell, M.-M. E.; Sheldon, A.; Tarsa, R. J.; Verni, C. J.; Coffen, D. L. Tetrahedron 1998, 54, 4051-4065.

10. (a) Hofmeier, H.; Schubert, U. S. Chem. Comm. 2005, 2423-2432; (b) Barberá, J.; Puig, L.; Romero, P.; Serrano, J. L.; Sierra, T. Chem. Mater. 2005, 17, 3763-3771; (c) Vollhardt, D.; Fainerman, V. B.; Liu, F. J. Phys. Chem. B 2005, 109, 11706-11711; (d) Arduini, M.; CregoCalama, M.; Timmerman, P.; Reinhoudt, D. N.; J. Org. Chem. 2003, 68, 1097-1106; (e) SauriatDorizon, H.; Maris, T.; Wuest, J. D.; Enright, G. D. J. Org. Chem. 2003, 68, 240-246 ; (f) Würthner, F.; Yao, J. Org. Chem. 2003, 68, 8943-8949 ; (g) Würthner, F.; Yao, S.; Heise, B.; Tschierske, C. Chem. Comm. 2001, 2260-2261; (h) Timmerman, P.; Prins, L. J. Eur. J. Org. Chem. 2001, 3191-3205, review ; (i) Goodman, A. J.; Breinlinger, E. C.; McIntosh, C. M.; Grimaldi, L. N.; Rotello, V. M. Org. Lett. 2001, 3, 1531-1534; (j) Löwik, D. W. P. M.; Lowe, C. 
R. Eur. J. Org. Chem. 2001, 2825-2839; (k) Li, X.; Chin, D. N.; Whitesides, G. M. J. Org. Chem. 1996, 61, 1779-1786.

11. (a) Iuliano, A.; Lecci, C.; Salvadori, P. Tetrahedron: Asymmetry 2003, 14, 1345-1353; (b) Iuliano, A.; Uccello-Barretta, G.; Salvadori, P. Tetrahedron: Asymmetry 2000, 11, 1555-1563.

12. Iuliano, A.; Voir, I.; Salvadori, P. J. Org. Chem. 1999, 64, 5754-5756.

13. (a) Lee, S. J.; Chang, J. Y. Tetrahedron Lett. 2003, 44, 7493-7497; (b) Muzikante, I.; Gerca, L.; Fonavs, E.; Rutkis, M.; Gustina, D.; Markava, E.; Stiller, B.; Brehmer, L.; Knochenhauer, G. Mater. Sci. Eng. 2002, C22, 339-343; (c) Lee, C.-H.; Yamamoto, T. Tetrahedron Lett. 2001, 42, 3993-3996; (d) Selby, T. D.; Stickley, K. R.; Blackstock, S. C. Org. Lett. 2000, 2, 171-174.

14. (a) Ren, S.; Fang, Q.; Lei, Y.; Chen, X.; Du, J. Cao, A. Macromol. Rapid Commun. 2005, 26, 998-1001; (b) Glaser, T.; Lügger, T.; Fröhlich, R. Eur. J. Inorg. Chem. 2004, 394-400; (c) Holst, H. C.; Pakula, T. Meier, H. Tetrahedron, 2004, 60, 6765-6775; (d) Steffensen, M. B.; Simanek, E. E. Org. Lett. 2003, 5, 2359-2361; (e) Meier, H.; Holst, H. C.; Oehlhof, A. Eur. J. Org. Chem. 2003, 4173-4180; (f) Yang, X.; Lowe, C. R. Tetrahedron Lett. 2003, 44, 1359-1362; (g) Xing, B.; Choi, M.-F.; Xu, B. Chem. Eur. J. 2002, 8, 5028-5031; (h) Hoog, P.; Gamez, P.; Driessen, W. L.; Reedijk, J. Tetrahedron Lett. 2002, 43, 6783-6786; (i) Verheyde, B.; Maes, W.; Dehaen, W. Mater. Sci. Eng. 2001, C18, 243-245; (j) Cook, S. Green Chem. 1999, G138-G141.

15. Thurston, J. T.; Dudley, J. R.; Kaiser, D. W.; Hechenbleikner, I.; Schaefer, F. C.; Holm-Hansen, D. J. Am. Chem. Soc. 1951, 73, 2981-2983; 2984-2986; 2986-2990.

16. (a) Pattarawarapan, M.; Reyes, S.; Xia, Z.; Zaccaro, M. C.; Saragovi, H. U. Burgess, K. J. Med. Chem. 2003, 46, 3565-3567; (b) Bork, J. T.; Lee, H.-S.; Chang, Y.-T. Tetrahedron. Lett. 2003, 44, 6141-6144; (c) Bork, J. T.; Lee, J. W.; Khersonsky, S. M.; Moon, H.-S.; Chang, Y.-T. Org. Lett. 2003, 5, 117-120; (d) Luo, G.; Xu, L.; Poindexter, G. S. Tetrahedron Lett. 2002, 43, 89098912; (e) Idem. Tetrahedron Lett. 2001, 42, 493-496; (f) Marsh, A.; Carlisle, S. J.; Smith, S. C. Tetrahedron Lett. 2001, 42, 493-496; (g) Falchi, A.; Taddei, M. Org. Lett. 2000, 2, 3429-3431; (h) Masala, S.; Taddei, M. Org. Lett. 1999, 1, 1355-1357; (i) Dijksman, A.; Arends, I. W. C. E.; Sheldon, R. A. Synlett 2001, 102-104; (j) Bigi, F.; Moroni, L.; Maggi, R.; Sartori, G. Chem. Comm. 2002, 716-717.

17. (a) De Luca, L.; Giacomelli, G.; Porcheddu, A. J. Org. Chem. 2002, 67, 5152-5155; (b) De Luca, L.; Giacomelli, G.; Porcheddu, A. Org. Lett. 2002, 4, 553-555; (c) Sandler, S. R. J. Org. Chem. 1970, 35, 3967-3968.

18. (a) Giacomelli, G.; Porcheddu, A.; Salaris, M.; Organic Lett. 2003, 5, 2715-2717; (b) Bandgar, B. P.; Pandit, S. S. Tetrahedron Lett. 2003, 44, 2331-2333; (c) Venkataraman, K.; Wagle, D. R. Tetrahedron Lett. 1979, 3037-3040.

19. Falorni, M.; Porcheddu, A.; Taddei, M. Tetrahedron Lett. 1999, 40, 4395-4396.

20. De Luca, L.; Giacomelli, G.; Porcheddu, A. J. Org. Chem. 2001, 66, 7907-7909.

21. (a) Rosenau, T.; Hofinger, A.; Potthast, A.; Kosma, P.; Org. Lett. 2004, 6, 541-544; (b) De Luca, L.; Giacomelli, G.; Porcheddu, A. J. Org. Chem. 2002, 67, 6272-6274.

22. Kaminska, J. E.; Kaminski, Z. J.; Góra, J. Synthesis 1999, 593-596.

23. Kaminski, Z. J.; Kolesinska, B.; Kaminska, J. E.; Góra, J. J. Org. Chem. 2001, 66, 6276-6281. 
24. (a) Díaz-Ortiz, A.; Elguero, J. Foces-Foces, C.; Hoz, A.; Moreno, A.; Moreno, S.; SánchezMigallón, Valiente, G. Org. Biomol. Chem. 2003, 1, 4451-4457; (b) Brewer, S. A.; Burnell, H. T.; Holden, I.; Jones, B. G.; Willis, C. R. J. Chem. Soc., Perkin Trans 2, 1999, 1231-1234 and references cited therein.

25. Kurteva, V. B.; Afonso, C. A. M. Green Chem. 2004, 6, 183-187.

26. Mathias, J. P.; Seto, C. T.; Simanek, E. E.; Whitesides, G. M. J. Am. Chem. Soc. 1994, 116, 17251736.

27. Hiroi, K.; Abe, J. Chem. Pharm. Bull. 1991, 39, 616-621.

28. Triethylamine was used instead of diisopropylethylamine.

29. Preparation of 2-imidazol-1-yl-ethanethiol (1): To a reflux solution of imidazole (9.002 g, 132.2 mmol) in anhydrous toluene $(120 \mathrm{~mL})$ was added a solution of ethylene sulfide $(2.6 \mathrm{~mL}, 44.0$ mmol) in anhydrous toluene (50 mL) during $21 \mathrm{hr}$ and was refluxed for more $2 \mathrm{hr}$. The solvent was evaporated in vacuo and the residue was diluted with diethyl ether (50 mL) and cooled to -6 ${ }^{\circ} \mathrm{C}$ (formation of solid). The mixture was filtered, washed the solid with $\mathrm{Et}_{2} \mathrm{O}(20 \mathrm{~mL})$ and the combined liquid fractions were evaporated to dryness and purified by distillation; b.p. 90 - $94{ }^{\circ} \mathrm{C} /$ $0.04 \mathrm{mmHg}$, to give 7.381 g of a 1.3:1.0 mixture (by ${ }^{1} \mathrm{H}-\mathrm{NMR}$ ) of 2-imidazol-1-yl-ethanethiol (l) and imidazole as a clear colourless oil; $v_{\max }\left(\right.$ film) $/ \mathrm{cm}^{-1} 3110,3037,2940,2840,2701,2616$, 1509, 1446, 1327, 1293, 1225, 1111, 1072, 1066, 929, 907, 827, 748, 663; $\delta_{\mathrm{H}}\left(400 \mathrm{MHz} ; \mathrm{CDCl}_{3}\right)$ 1.36 (1H, br s, SH), 2.87 (2H, t J 6.6, $\left.\mathrm{CH}_{2} \mathrm{~S}\right), 4.15$ (2H, t J 6.6), 6.96 (1H, s), $7.11(1 \mathrm{H}, \mathrm{s}), 7.55$ $(1 \mathrm{H}, \mathrm{s}) ; \mathrm{m} / \mathrm{z}(\mathrm{EI}) 128\left(\mathrm{M}^{+}\right), 101,81,69,61$; HRMS calcd. for $\mathrm{C}_{5} \mathrm{H}_{8} \mathrm{~N}_{2} \mathrm{~S}: 128.0408$, found: 128.0410 .

30. Ethyl 11-aminoundecanoate [31] (1 eq), triethylamine (1 eq) in 1,2-dichloroethane (reflux, 24 hr) was used.

31. Preparation of ethyl 11-aminoundecanoate (y): To a suspension of 11-aminoundecanoic acid $(0,017 \mathrm{~mol}, 3.5 \mathrm{~g})$ in absolute ethanol $(60 \mathrm{~mL})$ was added concentrated sulphuric acid $(0.020 \mathrm{~mol}$, $1.12 \mathrm{~mL}$ ). The resulting white solution was heated at reflux overnight, cooled $0^{\circ} \mathrm{C}$ and neutralized with aqueous saturated bicarbonate solution. The precipitated product was then collected by filtration, washed with cold water, and recrystallized from aqueous ethanol to give ethyl 11aminoundecanoate (y, 2.4g, 58 \%), m. p. $65^{\circ} \mathrm{C}$; $v_{\max }(\mathrm{NaCl}) / \mathrm{cm}^{-1}$; 3332, 2924, 2854, 1736, 1566, 1473, 1180; $\delta_{\mathrm{H}}\left(400 \mathrm{MHz} ; \mathrm{DMSO}_{6}\right) 1.15\left(3 \mathrm{H}, \mathrm{t}, \mathrm{J}=7.1 \mathrm{CO}_{2} \mathrm{CH}_{2} \mathrm{CH}_{3}\right), 1.22(12 \mathrm{H}, \mathrm{s}$, $\mathrm{NH}_{2} \mathrm{CH}_{2} \mathrm{CH}_{2}\left(\mathrm{CH}_{2}\right)_{6} \mathrm{CH}_{2} \mathrm{CH}_{2} \mathrm{CO}_{2} \mathrm{Et}$ ), 1.31 (2H, br, $\left.\mathrm{CH}_{2} \mathrm{CH}_{2} \mathrm{CO}_{2} \mathrm{Et}\right), 1.48$ (2H, br, $\mathrm{NH}_{2} \mathrm{CH}_{2} \mathrm{CH}_{2}$ ), 2.24 (2H, br, $\left.\mathrm{CH}_{2} \mathrm{CO}_{2} \mathrm{Et}\right), 2.86\left(2 \mathrm{H}, \mathrm{d}, \mathrm{J}=4.04, \mathrm{NH}_{2} \mathrm{CH}_{2}\right), 4.02\left(2 \mathrm{H}, \mathrm{q}, \mathrm{J}=7.1,-\mathrm{COOCH}_{2} \mathrm{CH}_{3}\right) ; \delta_{\mathrm{H}}$ (400 MHz; $\mathrm{CDCl}_{3}$ ) 1.20-1.24 (15H, m, $\mathrm{NH}_{2} \mathrm{CH}_{2} \mathrm{CH}_{2}\left(\mathrm{CH}_{2}\right)_{6} \mathrm{CH}_{2} \mathrm{CH}_{2} \mathrm{CO}_{2} \mathrm{CH}_{2} \mathrm{CH}_{3}$ ), 1.45 (2H, br, $\mathrm{CH}_{2} \mathrm{CH}_{2} \mathrm{CO}_{2} \mathrm{Et}$ ), 1.58 (2H, br, $\mathrm{NH}_{2} \mathrm{CH}_{2} \mathrm{CH}_{2}$ ), 2.25 (2H, t, J= 8, $\left.\mathrm{CH}_{2} \mathrm{CO}_{2} \mathrm{Et}\right), 2.67$ (2H, t, J= 8, $\left.\mathrm{NH}_{2} \mathrm{CH}_{2}\right), 4.09\left(2 \mathrm{H}, \mathrm{q}, \mathrm{J}=8, \mathrm{CO}_{2} \mathrm{CH}_{2} \mathrm{CH}_{3}\right) ; \delta_{\mathrm{C}}\left(\mathrm{CDCl}_{3}\right)$ 14.18, 24.90, 26.77, 29.06, 29.17, 29.31, 29.44, 34.30, 41.34, 60.05, 173.78; $\mathrm{m} / \mathrm{z}$ (CI) $230\left(\mathrm{MH}^{+}\right)$, 228, 184 ; EIMS calcd. for $\mathrm{C}_{13} \mathrm{H}_{28} \mathrm{NO}_{2}$ : 230.211, found: 230.212 .

32. In the case of the preparation of 1,3,5-triazines containing the dimethylamino group dimethylammonium chloride (1.0 mol eq.) and diisopropylethylamine (3.2 mol eq.) were used.

33. Preparation of (2-methoxyethyl)methylamine (j): A stirred solution of anhydrous triethylamine (5.0 mL, $53.63 \mathrm{mmol})$, 2-(methylamino)ethanol (4.028 g, $53.63 \mathrm{mmol})$, tetrabutylammonium 
bromide (432 mg, $1.34 \mathrm{mmol}$ ) and benzyl chloride (4.1 mL, $53.63 \mathrm{mmol}$ ) in anhydrous benzene $(40 \mathrm{~mL})$ was refluxed for 2 days under argon. The mixture was partitioned between $\mathrm{Et}_{2} \mathrm{O}(70 \mathrm{~mL})$ and aqueous sodium carbonate $(50 \mathrm{~mL})$. The aqueous phase was extracted with diethyl ether $(2 \mathrm{x}$ $70 \mathrm{~mL})$, the combined organic layers were dried $\left(\mathrm{MgSO}_{4}\right)$, filtered, evaporated to dryness and purified by distillation; b.p. 86 - $90{ }^{\circ} \mathrm{C} / 0.05 \mathrm{mmHg}$, to give 2-(benzylmethylamino)ethanol (e, $4.654 \mathrm{~g}, 79$ \%) as an colorless liquid; $v_{\max }(\mathrm{film}) / \mathrm{cm}^{-1} 3406(\mathrm{OH}), 3099,3076,3042,2957,2889$, 2855, 2804, 1503, 1458, 1372, 1077, 1032, 742, 702; $\delta_{\mathrm{H}}\left(400 \mathrm{MHz} ; \mathrm{CDCl}_{3}\right) 2.22$ (3H, s, NMe), 2.59 (2H, t J 5.2, 2-H), 2.86 (1H, br s, OH), 3.56 (2H, s, $\left.\mathrm{CH}_{2} \mathrm{Ph}\right), 3.62$ (2H, t J 5.2, 1-H), 7.287.34 (5H, m, Ph); m/z (FAB) $166\left(\mathrm{MH}^{+}\right), 134,120,107$; HRMS calcd. for $\mathrm{C}_{10} \mathrm{H}_{16} \mathrm{NO}$ : 166.1232, found: 166.1240 . To a solution of the above amino-alcohol $(1.364 \mathrm{~g}, 8.25 \mathrm{mmol})$ in anhydrous THF (60 mL) at room temperature under argon was added sodium hydride (396 mg, $50 \%$ oil dispersion, $8.25 \mathrm{mmol})$. After $15 \mathrm{hr}$ was added iodomethane $(0.51 \mathrm{ml}, 8.25 \mathrm{mmol})$, stirred for $8 \mathrm{hr}$ and refluxed for $1.5 \mathrm{hr}$. The mixture was partitioned between $\mathrm{Et}_{2} \mathrm{O}(50 \mathrm{~mL})$ and saturated aqueous ammonium chloride $(70 \mathrm{~mL})$. The aqueous phase was extracted with $\mathrm{Et}_{2} \mathrm{O}(2 \times 70 \mathrm{~mL})$, the combined organic layers were dried $\left(\mathrm{MgSO}_{4}\right)$, filtered, evaporated to dryness and chromatographed on a silica gel column (1:0 to 6:4 hexane/ethyl acetate) to afford benzyl-(2methoxyethyl)methylamine (1.159 g, $78 \%$ ) as a slightly yellow liquid; $v_{\max }(\mathrm{film}) / \mathrm{cm}^{-1} 3076$, 3042, 2985, 2940, 2883, 2849, 2815, 2798, 1503, 1458, 1372, 1123, 1077, 1054, 742, 702; $\delta_{\mathrm{H}}$ (400 MHz; $\left.\mathrm{CDCl}_{3}\right) 2.26$ (3H, s, NMe), 2.60 (2H, t J 5.8, 2-H), 3.34 (3H, s, OMe), 3.51 (2H, t J 5.8, 2-H), 3.55 (2H, s, $\mathrm{CH}_{2} \mathrm{Ph}$ ), 7.26-7.32 (5H, m, Ph); m/z (FAB) $180\left(\mathrm{MH}^{+}\right), 166,154,138,134$, 120; HRMS calcd. for $\mathrm{C}_{11} \mathrm{H}_{18} \mathrm{NO}$ : 180.1388, found: 180.1380. A mixture of the above compound (198.9 mg, $1.11 \mathrm{mmol})$ and $\mathrm{Pd} / \mathrm{C}(10 \%)(73 \mathrm{mg})$ in ethanol $(6 \mathrm{~mL})$ under hydrogen atmosphere (balloon) was strongly stirred at room temperature during $1.5 \mathrm{hr}$ (complete reaction by TLC). Argon atmosphere was introduced and the mixture was used directly to the next step.

34. In the other examples, pure amines were added to the reaction mixture.

Sample Availability: Available from the authors.

(C) 2006 by MDPI (http:www.mdpi.org). Reproduction is permitted for noncommercial purposes. 